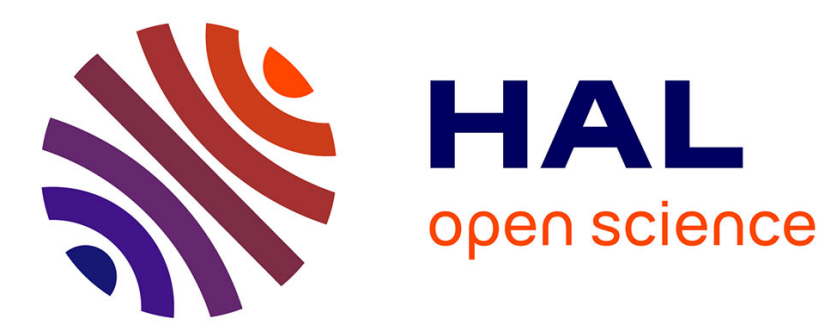

\title{
Recherches archéologiques en Gaule en 1944-1945
}

Raymond Lantier

\section{To cite this version:}

Raymond Lantier. Recherches archéologiques en Gaule en 1944-1945. Gallia - Fouilles et monuments archéologiques en France métropolitaine, 1947, 5 (1), pp.197-227. 10.3406/galia.1947.2036 . hal01919398

\section{HAL Id: hal-01919398 \\ https://hal.science/hal-01919398}

Submitted on 4 Feb 2020

HAL is a multi-disciplinary open access archive for the deposit and dissemination of scientific research documents, whether they are published or not. The documents may come from teaching and research institutions in France or abroad, or from public or private research centers.
L'archive ouverte pluridisciplinaire $\mathbf{H A L}$, est destinée au dépôt et à la diffusion de documents scientifiques de niveau recherche, publiés ou non, émanant des établissements d'enseignement et de recherche français ou étrangers, des laboratoires publics ou privés.

\section{(ㅇ)(1) $\$$}

Distributed under a Creative Commons Attribution - NonCommercial - NoDerivatives 44.0 


\section{CHRONIQUE DES PUBLICATIONS}

\section{RECHERGHES ARCIIÉOLOGIQUES EN GAULE EN 1944-1945¹}

\section{GÉnÉRalités}

L'histoire des recherches archéologiques en France s'est enrichie d'un certain nombre de travaux. Le marquis de Luppé2, utilisant les volumes parus de la correspondance de Prosper Mérimée, apporte quelques précisions à l'étude du laborieux et consciencieux inspecteur général des Monuments Historiques. Ce que ne disent pas les Notes de voyage apparait dans les lettres échangées avec les archéologues, dont il a fait connaissance au cours de ses tournées. Il les conseille, les guide, leur rend de menus services. Plus que ses écrits techniques, ces documents témoignent de tout ce que notre archéologie doit à Prosper Mérimée. Le livre qu'A. Grenier ${ }^{3}$ a consacré à l'attachante figure de Camille Jullian est aussi l'histoire du grand mouvement universitaire qui, après les désastres de 1870 , a provoqué le relèvement de notre enseignement supérieur. L'œuvre de Jullian est inséparable du temps où il

(1) Voir Gallia, 1, 1942, p. 189-209; 2, 1943, p. $228-263 ; 3,1944$, p. $263-292 ; 4,1946$, p. $320-353$.

(2) Mérimee. Paris, A. Michel, 1945, in $-8^{\circ}$, p. 75-94.

(3) Camille Jullian. Un demi-siècle de science historique et de progrès français, 1880-1930. Paris, A. Michel, 1944, in- $8^{\circ}$. a vécu. Éminent historien, il fut aussi un grand Français.

Le centenaire de la Revue archéologique et le vingtième anniversaire de la fondation de la Société des Etudes latines ${ }^{5}$ ont été l'occasion de mémoires retraçant l'œuvre accomplie par ces organismes dans la recherche et l'étude de nos antiquités nationales.

Parmi les bibliographies, on accueillera avec une particulière reconnaissance la reprise, par $M$. René Gandilhon ${ }^{6}$, de l'œuvre, trop longtemps interrompue, des Lasteyrie, Lefebvre-Pontalis et Vidier. La présentation n'a pas changé et la méthode ne diffère pas sensiblement de celle adoptée pour les précédents volumes, si ce n'est qu'un certain nombre d'améliorations ont été apportées : place plus importante faite aux travaux de géographie et de folklore; développement

(4) R. Lantier, Autour d'un centenaire (18441944), dans Reu. archéol., 1945, 1, p. 5-25.

(5) A. Grenier, Les etudes gallo-romaines (1923-1943), dans Mémorial des Etudes latines, publié à l'occasion du $20^{\circ}$ anniversaire de la Societé des Etudes latines, 1943, p. 609-627. Ch. Picard, Vingt ans d'études sur les arts de l'Italie antique, ibid., p. 500-605.

(6) Bibliographie générale des travaux historiques et archéologiques publies par les sociétés savantes de la France, dressée sous les auspices du Ministère de l'Éducation nationale, sous la direction de Ch. Samaran. Période 1910-1940. T. I, Ain-Creuse. Paris, Impr. nat., 1944, in-4º. 
de l'analyse des comptes rendus des séances des sociétés savantes; relevé plus complet des notices nécrologiques. Les Annales de Bourgogne ${ }^{1}$ et le Bulletin trimestriel de la Société des Letlres, Sciences et Arts du département de la Lozère ${ }^{2}$ ont continué la publication de leurs bibliographies régionales.

Les livrets d'histoire locale ${ }^{3}$, sous la direction de Jacques de Font-Réaulx, apportent les notions indispensables de géographie historique et administrative, les principaux faits d'histoire, d'archéologie et de folklore, avec mention des sources, ouvrages, revues et monographies communales. Leur domaine s'étend de la préhistoire à la période contemporaine. Dans les petites monographies provinciales, publiées dans la collection Que sais-je?4, on trouvera un résumé liminaire de l'histoire du pays dans l'antiquité. Avec la collection Connais ton pays, que dirige A. Grenier, on se propose de mettre à la portée du public les notions les plus récemment acquises sur la vie, les mours, les industries, les traditions de nos ancêlres. Deux volumes ont paru traitant de l'anthropologie des Français ${ }^{5}$ et de la formation du peuple

(1) T. XVII, 1945, p. 283-288 : Pierre Gras, Bibliographie bourguignonne. I. Epoques préhislorique, gauloise, gallo-romaine, burgonde et tranque.

(2) 1944, p. 495-505, 553-574 : Marius BALmelle, Bibliographie du Gévaudan. Anthropologie, préhistoire, protohistoire. La race lozérienne des Baumes-Chaudes.

(3) I. Manuel des ètudes dromoises, par J. DE Font-RÉaulx; II, Manuel des éludes vivaraises, par lílie Rrynier; Manuel des études héraultaises, par ĺmile Appolıs; IV, Manuel des études bourbonnaises, par Joseph VIPLE. Valence, Imprimeries réunies, 19411945,4 vol. in-16, avec cartes.

(4) Henri WaQuET, Histoire de la Bretagne, 1943 ; René Rigodon, Histoire de l'Auvergne, 1944 ; H. Avezou, Histoire de la Savoie, 1944.

(5) H. V. Valloors, Anthropologie de la popu- gaulois, ainsi que des vicissitudes de la Gaule jusqu'à la conquête romaine ${ }^{6}$.

Une nouvelle, quelque peu inattendue, nous vient de Montpellier : la création, à la Faculté des Lettres de l'Université, d'un certificat d'études préhistoriques, valable pour la licence ès lettres ${ }^{\text {? }}$. Quelles seront maintenant les autres Facultés des Lettres qui suivront l'exemple de Montpellier? Finira-t-on par comprendre que notre histoire nationale commence avec les temps paléolithiques? Ln "Centre d'études bourguignonnes" est crèé près de la Faculté des Lettres de Dijon $^{8}$, et la Commission des Antiquités de la Côte-d'Or ${ }^{9}$ se préoccupe de l'établissement de répertoires sur les grands problèmes archéologiques (voies romaines, monuments religieux gallo-romains, cimetières barbares). Publié sous les auspices de la Commission des fouilles archéologiques, le manuel d'Édouard Salin ${ }^{10}$ doit être le livre de chevet de tous les fouilleurs, tant ses conseils sont pertinents et peuvent s'appliquer à la fouille de toute sépulture à quelque époque qu'elle appartienne. On lira également avec profit les conclusions de la conférence tenue à Londres, en 1943, sur l'avenir de l'archéologie ${ }^{11}$. Signalons

lation française. Paris-Toulouse, Didier, 1943, petit in $-4^{\circ}$.

(6) Albert Grenier, La Gaule celtique. ParisToulouse, Didier, 1945, petit in- $4^{\circ}$.

(7) Un arrêté du 19 janvier 1945 précise les modalités de l'examen.

(8) Annales de Bourgogne, 17, 1945, p. 66-67.

(9) Ibid., 17, 1945, p. 136-137.

(10) Manuel des fouilles archéologiques. 1. fidouard Salin, Les fouilles de sépullures du $V^{\bullet}$ au VII ${ }^{\circ}$ siecle. Paris, Les Presses universitaires de France, 1946, in- 16.

(11) Universily of London, Inslitute of Archaeology. Conference on the Fulure of Archaeology, held at the University of London, Institute of Archaeology, August 6th. to 8th. 1943. Londres, The University, 1943 , in- $4^{\circ}$. 
la publication d'un nouveau bulletin archéologique, édité par la Société préhistorique du Languedoc ${ }^{1}$, et une revue technique qui se propose de traiter des métaux dans l'histoire, les techniques et les arts, depuis la préhistoire jusqu'à nos jours ${ }^{2}$.

\section{Palíolithique et Mísolituique}

Ce n'est pas un manuel de préhistoire que donne Mlle $\mathrm{H}$. Alimen ${ }^{3}$ qui a terminé l'ouvrage laissé inachevé par Léonce $\mathrm{J}_{0}$ leaud. Il s'agit de l'étude du milieu physique dans lequel vécut l'Homme paléolithique : géologie du Quaternaire et ses méthodes ; chronologie relative et absolue; milieux glaciaires, fluviatiles, lœssiques, marins, provinces biologiques. Une seconde partie traite des Primates et de l'Homme.

La Société d'études des sciences naturelles du Gard a assumé la publication posthume du mémoire présenté pour le diplôme d'études supérieures par JeanneUlysse Dumas ${ }^{4}$, qui constitue un bon répertoire des stations paléolithiques du département. Le Paléolithique ancien est représenté par des gisements de plein air et des remplissages de cavernes moustériens; le Paléolithique supérieur par un matériel lithique magdalénien, assez difficile à déterminer et par une industrie très pauvre de l'os. On a

(1) Bulletin de la Société préhistorique du Languedoc (rédaction et administration, rue Malaret, 25, Toulouse, Haute-Garonne).

(2) Metaux et civilisation.

(3) Léonce Joleaud et Henriette Alimen, Les temps préhistoriques. Bibliothèque de Philosophie scientiflque. Paris, Flammarion, 1945, petit in-4.

(4) Jeanne-Ulysse Dumas, La Préhistoire du Gard. Le Quaternaire, Société d'Étude des sciences naturelles du Gard. Nimes, Bruguier, 1944 , in-8. parlé d'Aurignacien final et de Solutréen, mais on peut se demander si ces industries ne représentent pas plutôt un Magdalénien très ancien. Au point de vue de l'art, les grottes à figures du département ne seraient que la continuation assez dégénérée de l'art de la Vézère. L'étude de la faune ${ }^{5}$ apporte quelques précisions à la reconstitution du paysage de la région pendant le Pléistocène supérieur, époque à laquelle apparaît une faune froide, tardive, installée dès le Moustérien moyen, avec tous ses éléments caractéristiques. Des changements se produisent, au cours de la dernière glaciation. L'Elephas primigenius et le Rhinoceros tichorhinus disparaissent dès le début du Paléolithique supérieur. Dans les gisements prédominent les restes de l'Equus caballus (Aurignacien), puis du Renne et du Bouquetin. Aucun élément caractérisitque de la faune steppique n'a été rencontré. Cette absence et la présence du Bouquetin contribuent à donner un faciès particulier à la faune du Midi méditerranéen, à la fin de la dernière glaciation.

L'excellent connaisseur des gisements corréziens $^{6}$ qu'est l'abbé J. Bouyssonie a dressé le tableau du Paléolithique dans cette région, particulièrement riche en stations préhistoriques. L'Abbevillien apparait sous la forme d'outillages isolés en quartz ou en silex, dans les vallées (de Maumont, au Griffolet; de la Corrèze, près de Malemort; de la Vézère, au Sail-

(5) A. Bonnet, Les faunes pleistocènes du Gard, dans B. S. P. F., XLII, 1945, p. 43-48.

(6) Abbé Jean Bouyssonie, La préhistoire en Correze, dans Bull. soc. scientif. hist. et archeol. Corrèze, LXV, 1944, p. 37-55. - Du même, Le gisement mousterien de "Chez Pourré , et de "Chez Comte ", près de Brive (Corrèze), dans Gallia, 3, 1944, p. 225-229; - du même, La grotte Dufour, près Brive (Correze), dans B. S. P. F., XLI, 1944, p. 186-192. 
lant) et sur les hauteurs, près de La Pigeonnie à Yssandon. Le Paléolithique moyen est mieux représenté par des instruments tenant à la fois de l'Acheuléen, du Moustérien, sur l'une et l'autre rive de la Corrèze, principalement sur la rive gauche, à Grandmont et à $\mathrm{La}$ Ramière, en passant par Montplaisir, à Puymège, à Noailles, gisements de plein air situés sur les hauteurs vers le Périgord. Des silex roulés ont été recueillis sur les terrasses alluviales de 30 à 25 mètres, ainsi que dans les rivières. Le climat est alors peu rigoureux, mais les fonds de vallées ne sont guère praticables. Avec le froid qui accompagne la glaciation würmienne, l'Homme s'installe dans les grottes. Deux de ces abris sont particulièrement importants, les gisements de "Chez Pourré " et de "Chez Comte " et La Chapelle-aux-Saints, où fut découvert un squelette néanderthaloïde. Dans les foyers noirâtres de la couche I de "Chez Comte " et "Chez Pourré ", on a ramassé des pointes de formes variées, des racloirs à bords épais ou minces, des disques, quelques burins, des "bolas ", en silex et en quartz. Dans l'horizon sableux II, il n'y a que des racloirs, quelques pointes et des éclats utilisés de quartz, de granit, de gneiss et de quartzites. Les limons argileux du troisième niveau ont donné un outillage moustérien et, à la surface, quelques pièces de type aurignacien, mais principalement des bifaces qui ont pu être entrainés par les ruissellements. Pendant le Paléolithique supérieur, les stations sont généralement groupées le long des ruisseaux descendant de Montplaisir, la Planche-Torte et ses affluents, la Couze, et dans les cavernes du Sud de Brive. L'industrie du silex est importée, les Paléolithiques ont utilisé le jaspe et les galets de rivière. L'Aurignacien typique est bien caractérisé à La Coumba-
del-Bouïtou et à Chanlat; la transition entre cette industrie et le Solutréen à Font-Yves, Bos-del-Ser, "Chez Serre ", à Noailles, à La Font-Robert, à la grotte des Morts; le Solutréen \& Pré-Aubert, Basdegoule, Puy-de-Lacan; le Magdalénien à Terrasson et dans la vallée de Planche-Torte. Pendant le Mésolithique, dont les industries tendent à se rapprocher de celles du Haut-Limousin, l'Homme a établi des campements de plein air sur les basses-terrasses des vallées. Au Néolithique, il s'installe sur les plateaux. Ses traces ont été retrouvées au voisinage des localités modernes les plus importantes, c'est-à-dire dans les régions les plus fertiles du département. Mais ses habitats sont clairsemés. L'occupation la plus dense de la Corrèze est contemporaine du Moustérien et du Périgordien.

La rareté du silex et de pierres faciles à tailler, l'éloignement des centres de production explique la rareté du Paléolithique en Bretagne ${ }^{1}$. Les quelques stations reconnues sont établies sur le littoral, à proximité des gisements de silex que donnent les plages soulevées, ou au voisinage des affleurements de quartzite à grain fin. C'est autour de ces gisements que l'on doit prospecter avec des chances de succès. Il semble que les stations bretonnes ont été occupées au cours de la troisième glaciation. Les outillages moustériens ont été recueillis dans le "head sableux ", couronnant l'ancienne plateforme émergée. Au Bois du Rocher, le Moustérien inférieur serait contemporain d'une émersion et le MontDol aurait été occupé à la fin du mou-

(1) P. R. Grot, Stations paléolithiques de Bretagne, dans B. S. P. F., XLI, 1944, p. 154155. - L. Coln.in, L'âge du Paléolithique du Nord el de l'Ouest de la Bretagne, dans Bull. soc. archéol. départ. Ille-et-Vilaine, XLVII, 1914, p. 31-38. 
vement d'émersion des plages soulevées monastiriennes.

En Gâtinais ${ }^{1}$, la vallée du Loing, pays de transit entre Loire et Seine, est jalonnée d'une trainée presque ininterrompue de stations préhistoriques. La région de Montargis, trop humide, est moins habitée, alors que les buttes sableuses des environs de Nemours ont livré des campements magdaléniens et lardenoisiens. Sur la rive droite, on relève des traces des diverses civilisations paléolithiques. Les Néolithiques ont préféré les plateaux fertiles et la proximité des sources.

A l'occasion de la publication de deux pointes en forme de laurier, conservées au château de La Guitière, à 2 kilomètres au Sud de Saint-Pierre-de-Maillé (Vienne), Et. Patte ${ }^{2}$ étudie la dispersion de l'industrie solutréenne dans la Vienne. Les gisements sont placés sur un étroit couloir, ménagé entre le Massif Central, les plaines, les plateaux voisins de l'Océan Atlantique, constituant une région de passage entre l'Aquitaine et l'Espagne. Dans les causses de l'Indre, aux falaises du Roc, dans le talus de l'Arcade, près d'un gisement aurignacien, on a constaté l'existence d'un petit ensemble moustérien de tradition acheuléenne, au confluent de la Creuse et de la Bouzanne. Des abris moustériens ont été aussi reconnus dans le département d'Indreet-Loire ${ }^{3}$, et dans la vallée de la Brenne, à la Bouyère, commune de Douadie (Indre), un biface moustérien ${ }^{4}$. Un inven-

(1) Abbé André Nouel, Contribution d la préhistoire du Gâtinais (Inventaire d'une collection detruite en 1940), dans B.S. P. F., XLIl, 1945, p. 100-102, 228-231.

(2) Le Solutréen dans la Vienne, dans B.S. P. F., XLI, 1941, p. 42-46.

(3) Olivier Сharbonnier, Les falaises des Roches (Indre), dans Revue de l'Acad. du Cenire, 70, 1944, p. 78-98.

(4) Du même, La Brenne (Indre) a-t-elle été taire des bifaces pour le département de Maine-et-Loire a été dressé par le $\mathrm{D}^{\mathrm{r}}$ Gruet $^{5}$, et à propos de la découverte d'un coup-de-poing moustérien à Villefranche-de-Rouergue, A. Cabrol ${ }^{6}$ donne la liste des découvertes paléolithiques faites dans l'Aveyron et qui ne sont pas nombreuses. En Limousin, un disque moustérien a été trouvé aux environs de Droux (Haute-Vienne) ${ }^{7}$, ainsi que des silex plutôt tardenoisiens.

Le gisement de Fontmaure $^{8}$ a fait connaitre des objets nouveaux en forme de tablettes à bord abattu, dans un niveau de la fin du Moustérien, influencé par les techniques des lames et lamelles du Paléolithique supérieur. L'industrie est à rapprocher de celles du niveau supérieur de La Quina et de la couche E de la Ferrassie. Les outils à encoches sont nombreux, comme dans les couches aurignaciennes. E. Passemard ${ }^{9}$ a donné un important mémoire consacré à la présentation des fouilles qu'il conduisit, de 1913 à 1922, dans la caverne d'Isturitz (Basses-Pyrénées). Dans le même département, à Labastide, dans une grotte, une cachette de grandes lames magdaléniennes a été mise au jour ${ }^{10}$. Les travaux de défense, organisés par les Allemands

habitée au Paléolithique? dans B. S. P. F., XLII, 1945, p. 93-96.

(5) Inventaire des bifaces trouvés en Maineet-Loire, ibid.. XLII, 1945, p. 199-200.

(6) A propos d'un coup de poing moustérien découvert da Villefranche-de-Rouergue (Aveyron), ibid., XLI, 1944, p. 164-165.

(7) J. Percier, Bull. soc. archéol. el hist. du Limousin, LXXXI, 1945, p. 56-59.

(8) Dr L. Pradel, Le gisement du Moustérien typique final da influences paléolithiques de Fontmaure (commune de Valleches, Vienne), dans B. S. P. F., XLII, 1945, p. 84-96. Voir ibid., XLI, 1944, p. 23-26.

(9) La caverne d'Isturitz, en Pays Basque, dans Préhistoire, IX, 1944.

(10) Glory et G. R. Simonet, Bull. soc. préhist. Languedoc, 1945, 402, p. 1. 
dans la banlieue de Toulouse ${ }^{1}$, ont fait connaitre des gisements de quartzites taillés, sur la terrasse de 30 mètres de la Garonne, niveau de Saint-Martin-duTouch, et dans le lit de l'Hers, entre les ponts de Lasbordes et de Montaudron. Les fouilles du cimetière moderne de Saint-Romans (Isère) ${ }^{2}$, établi sur une nappe de graviers, contemporains de la décrue de la dernière glaciation, ont précisé que la station magdalénienne était plus récente que le dépôt des graviers et que peu de temps s'était écoulé entre le retrait de la dernière glaciation et l'installation des Magdaléniens. Les gisements des grottes et abris sous roches de Thônes (Puy-de-Dôme) sont contemporains de la fin des temps paléolithiques et leur industrie est semblable à celle recueillie à Chaix ${ }^{3}$. Sur la haute basse-terrasse de Montières (Somme), à Boutmy, a été signalée la présence de bifaces façonnés selon la technique du Levalloisien ancien par enlèvement d'éclats'.

Avant l'arrivée des Magdaléniens en Périgord, on constate à Laugerie-Haute, dans le niveau sous-jacent au Solutréen ancien, une évolution artistique et industrielle tendant déjà vers le Magdalénien ${ }^{5}$. Le même fait se produit dans l'Est de la France, à La Colombière (Ain), et jusqu'en Moravie, à Vistoniče. Dès le Périgordien final, en dehors de la région

(1) L. MÉroc, B. S. P. F., XLII, 1945, p. 124.

(2) Frank Bourdier et Marc Millah, Nouvelles fouilles du gisement préhistorique du cimetière de Saint-Romans (Isère), dans P.-V. soc. scientif. Isère, $\mathrm{n}^{\circ} 158,4$ mars 1941; extr. $4 \mathrm{p}$.

(3) G. Desrut et E. Deret, Les grottes el abris préhistoriques de Thônes (Puy-de-Dôme), dans B. S. P. F., XLI, 1944, p. 34-38.

(4) R. P. H. FleisGh, B. S. P. F., XLI, 1944, p. 165-167 ; XLII, p. 135-137.

(5) D. Peyrony, Origine du Magdalénien I $\dot{a}$ "éclats de silex $\grave{a}$ retouches abruptes, dans B. S. P. F., XLI, 1944, p. 127-129. pyrénéenne, les éclats de silex à retouches abruptes abondent dans les niveaux directement superposés au Sclutréen supérieur, en Dordogne, Charente, Corrèze, Seine-et-Oise, Seine-et-Marne, Loiret, mais ils n'atteignent ni le Sud de la Garonne, ni le Midi. On en conclut que le plus ancien Magdalénien du Périgord et de la Charente serait parvenu dans ces régions du Massif Central, repoussé par les porteurs de la civilisation du M. II à triangles scalènes, venant du Nord-Est. Ils y auraient vécu jusqu'à l'extension vers le Nord des Madgaléniens pyrénéens.

Deux inventaires sont consacrés aux lampes paléolithiques ${ }^{6}$ et aux sphéroïdes moustériens d'Artois et d'Ile-de-France ${ }^{7}$.

$\mathrm{Au}$ sujet des squelettes magdaléniens du Cap-Blanc et de Saint-Germain-laRivière, D. Peyrony ${ }^{8}$ fait observer qu'ils ont été trouvés couchés sur le côté gauche, les membres inférieurs repliés, orientés Est-Ouest, la tête vers le Levant. Ces dispositions sont caractéristiques de prescriptions rituelles et ne correspondent aucunement à un écrasement, comme l'avait supposé Testut.

Peu de découvertes sont à signaler dans le domaine de l'art du paléolithique supérieur : des gravures du M. II à Crabillat et à Laugerie-Haute ${ }^{9}$, à la grotte des Fées à Marcamps (Gironde) ${ }^{10}$; tracés au doigt aurignaciens à la grotte d'Aldène (Hérault); tête d'Ours sur argile avec oreille modelée en relief, à la

(6) Dr A. H. Bastin, ibid., XLII, 1945, p. $34-38$.

(7) H. Lambert, Sphéroides moustériens d'Artois et d'Ile-de-France, dans B. S. P. F., XLI, 1944, p. 177.

(8) D. Peyrony, L'Homme magdalénien de Laugerie-Basse a-t-il êté écrasé? dans Bull. Soc. hist. et archéol. Périgord, LXXIII, 1945, p. 23-24.

(9) D. et E. Peyrony, B. S. P. F., XLII, 1945, p. 29-30.

(10) H. Lambert, ibid., XLII, 1945, p. 140 143. 
grotte de Peyort, près de Prat (Ariège) ${ }^{1}$. On lira avec un amusement attristé les souvenirs du Comte H. Begouen sur l'affaire du bas-relief de Laussel, acquis frauduleusement par le Musée de Berlin en 1913. "Surtout pas d'histoires!", clama le ministre des Affaires étrangères de cet heureux temps. J. Vézian ${ }^{3}$ décrit les peintures et les œuvres d'art mobilier de la grotte du Portel.

Il faudrait abandonner le terme pourtant commode et consacré par l'usage de Mésolithique, mais l'auteur ${ }^{4}$ ne propose pas de solution acceptable. En archéologie, il n'y a pas que des classements typologiques et les mots paléolithique, mésolithique, néolithique, ont un contenu bien plus étendu que le rappel d'un facies industriel. Ils correspondent à un ensemble de civilisations. Pourquoi vouloir encombrer la nomenclature archéologique de noms nouveaux, sous le prétexte qu'il est des pointes mésolithiques qui ne correspondent à aucune forme géométrique $^{5}$ ? Il y aura bientôt autant de types de pointes que de marques d'automobiles, de coutellerie ou de porcelaine.

Une définition précise est donnée du mot alios ${ }^{6}$ : couche plus ou moins noire, provenant de la décomposition des déchets de cuisine, des cendres de foyers et des fumiers humains, consolidés par imprégnation d'oxyde de fer, opération facilitée par les abris élevés par l'Homme.

(1) Abbé Glory, ibid., XLI, 1944, p. 151-153.

(2) Ibid., XLII, 1945, p. 182-183.

(3) Bull. soc. préhist. Languedoc, n० 2, 1945, p. $2-11$.

(4) J. Blanchard, La question du Mésolithique el le classement des industries paleolithiques, dans B. S. P. F., XLII, 1945, p. 31-34.

(5) L. Coutier, J. Blanchard et E. Vignard, Les pointes de Sonchamp (Seine-et-Oise), ibid., XL1I, 1945, p. 130-134.

(6) E. Giraud et E. Vignard, L'alivs spécial des gisements tardenoisiens de la région parisienne, ibid., XLI, 1944, p. 108-112.
L'alios est la limite extrême sur laquelle sont déposés les objets de l'industrie mésolithique.

Sur les fouilles de Blangy-sur-Bresle et le Campignien, on se reportera avec profit au mémoire de M. Duteurtre?

L'exploration des gisements mésolithiques de la forêt de Montmorency ${ }^{8}$ continue à donner des résultats intéressants. Dans la vallée sèche, qui descend du plateau de Blémur vers l'église de Piscop, et à la batterie de Blémur, a été découverte une industrie de pointes, perçoirs, pics, retouchoirs, burins, pointes-burins, tranchets, grattoirs, obtenus par débitage par percussion sur bloc brut, sans plan de frappe préparé. Sur l'épine dorsale d'une petite butte, entre le ruisseau des Vaux-de-Cernay et la route des Vaux à Auffargis, un abri de chasse a donné une industrie tardenoisienne avec pointes sauveterriennes scalènes (Sauveterrien II) et pointes à cran $^{9}$. Dans la haute vallée de l'Essonne ${ }^{10}$, le Paléolithique est très peu abondant. La région a été occupée principalement par les Tardenoisiens, puis par les Néolithiques. Il en est de même pour le bassin d'Aurillac ${ }^{11}$.

Le principal intérêt des fouilles conduites dans l'abri sous roche situé à l'entrée de la grotte de Cuzoul, à 44 mètres au Sud-Ouest de Gramat ${ }^{12}$, a

(7) Ibid., XLII, 1945, p. 62-64.

(8) A. Bogard, Un faciès nouveau de la forèt de Monimorency, ibid., XLI, 1944, p. 27-32.

(9) R. Robert et E. Vignard, Les campements mésolithiques du "Désert d'Auffargis * (Seine-et-Oise), ibid., XLII, 1945, p. 76-84.

(10) Abbé R. Moufflet, La préhistoire dans la haute vallée de l'Essonne, ibid., XLII, 1945, p. 223-227.

(11) R. Pierron, Civilisations lithiques du bassin d'Aurillac, dans Revue de la HauteAuvergne, XXX, 1944, p. 241-259.

(12) R. Lacam, A. Niederlander, H. V. ValLoIs, Le gisement mésolithique du Cuzoul de 
été l'établissement d'une chronologie pour les établissements mésolithiques du Haut-Quercy. La stratigraphie de la station apporte également la justification des subdivisions, proposées par M. Coulonges pour le Tardenoisien, et la complète même sur certains points. L'évolution du Mésolithique au Cuzoul peut ainsi s'établir : Sauveterrien évolué et Postazilien évolué : niveau II du Martinet et niveau I du Cuzoul; Tardenoisien I typique, réminiscences paléolithiques, pointes tardenoisiennes typiques ; niveau III du Martinet et niveau II du Cuzoul ; apparition de la pointe à base récurrente au Cuzoul III ; - Tardenoisien II : apparition des pointes tardenoisiennes au Cuzoul IV; - Tardenoisien III, prédominance des petits tranchets; au Cuzoul IV, apparition de la technique néolithique ; fin du Mésolithique, Tardenoisien III évolué ; pointes à pédoncule, pointes-hamegons, poteries, sculptures au Martinet II. Les fouilles ont apporté peu de précisions sur les modes de vie pendant le Mésolithique. La grotte ayant été à peine occupée, on pcut supposer un habitat en cabanes. La population, peu nombreuse et pauvre, tire ses principales ressources de la chasse (Cerf, Sanglier, Cheval) et utilise, pour la fabrication de son outillage, les roches les plus diverses : silex du Sarladais it de l'Agenais, et même le mauvais calcaire siliceux local. Les périodes d'abandon de l'ab̉ri correspondent à des déplacements temporaires, peut-être en direction de la station du Martinet, située à quelques jours de marche. Ainsi expliquerait-on la présence, dans l'un et l'autre gisement, de certains types industriels, telles les pointes à base récurrente. Toutefois l'évolution progressive de

Gramat. Archives de l'Institut de Paléontologie humaine, mém. no 21. Paris, Masson, 1944, in-4º. l'outillage, au Cuzoul, témoigne de la fidélité des occupants à leurs demeures. Il n'y a pas de céramique dans le gisement. La découverte, dans le deuxième niveau, d'un squelette d'homme, en plein horizon tardenoisien typique, prouve l'existence d'un culte des morts à cette époque. Le cadavre avait été étendu sur le dos, les jambes et les bras allongés, la face tournée vers l'Est, la tête reposant sur un assez gros bloc de calcaire siliceux, intentionnellement taillé. L'Homme du Cuzoul se rattache au type dolichomésocéphale, représenté à Ofnet, Téviec et Montardit. D'autres caractéristiques le rapprochent des Hommes de Mugem. Il apparait "comme un intermédiaire entre les deux grands groupes d'Hommes mésolithiques de l'Europe occidentale, fait d'autant plus intéressant que le gisement de Gramat se trouve justement à mi-distance entre ceux de Téviec et Ofnet au Nord, celui de Mugem au Sud $n$ [H. V. Vallois].

Dans les régions de Terrasson et de Cublac, les gisements du Tardenoisien moyen et du Néolithique ont livré des flèches tranchantes triangulaires, trapézoïdales et à talon ${ }^{1}$. En Argonne meusienne $^{2}$, les vallées de l'Aire et de la Biesme, au vallon de Natifontaine et aux cinq sources du Claon, ont été occupées par des Tardenoisiens.

\section{Néolithique et Age du Bronze}

Les dernières découvertes mésolithiques du Périgord apportent quelques clartés sur l'histoire du Néolithique dans

(1) $\mathrm{D}^{\mathrm{r}} \mathrm{A}$. Cheynier, Les flèches tranchantes dans le Terrassonnais, dans Bull. soc. scientif., hist. el archéol. Corrèze, 66, 1944, p. 56-60.

(2) G. Chenet, De quelques stationnements tardenoisiens en Argonne meusienne, dans B. S. P. F., XLII, 1945, p. 96-97. 
la province'. On assiste d'abord à l'expansion progressive des Aziliens qui occupent peu à peu les abris. Mais ils n'ont pas séjourné longtemps dans les vallées de la Dordogne, de la Vézère et de l'Isle, alors que dans celle de la Droune, à Rocheraillé, ils ont laissé d'importants dépôts dont certains sont contemporains du néolithique. Les Tardenoisiens ont chassé les Aziliens du centre de la Dordogne et ils ont survécu à l'invasion néolithique, qui semble avoir été assez tardive. Leurs établissements à la Roque-Saint-Christophe, aux Marseilles, à Laugerie-Haule appartiennent à la phase récente du Néolithique. Dans le Bergeracois, les tranchets et les haches taillés en silex ne représentent pas une industrie campignienne, car aucun vestige du passage des Campigniens en Limousin n'a encore été retrouvé. Il s'agit d'un Néolithique à influence campignienne.

Dans le bassin parisien, le couloir du Loing a été la route suivie par les Néolithiques pour pénétrer dans la région comprise entre Loire et Seine. Pendant le Campignien, les stations-ateliers et les campements y sont nombreux. Au Néolithique moyen, on assiste au garnissage du rebord des plateaux, et à la descente dans les vallées au Néolithique récent. Le problème de l'eau, l'existence de terres légères, faciles à mettre en culture, ont joué un rôle important dans le régime de l'occupation à cette époque ${ }^{2}$. Les établissements néolithiques du Bois de Clamart ${ }^{3}$ ont fourni un outillage

(1) D. Peyrony, Le Néolithique en Périgord, extr. du Bull. Soc. hist. et archéol. du Périgord, 1944, 6 p.

(2) L. Nougier, B. S. P. F., XLII, 1945, p. 10-12, 25-26.

(3) R. Daniel, Gisemenis néolithiques du Bois de Clamarl (Seine), ibid., XLI, 1944, p. 51-58. misérable, appartenant à une période assez récente du groupe Seine-OiseMarne. En Seine-et-Oise, aux Roches ${ }^{4}$, quelques abris ont été occupés depuis le Néolithique jusqu'au nI $^{\mathrm{e}}$ siècle de notre ère, dispersés dans les éboulis. Malgré un titre prometteur, il y a peu de renseignements précis à retirer des articles de R. Pierron ${ }^{5}$ sur le peuplement pré- et protohistorique de la région cantalienne. En Touraine, un campement temporaire de chasse a été découvert à la Fontainedu-Saule, en forêt d'Amboise, sur le plateau situé entre Loire et Cher ${ }^{6}$. Dans la banlieue de Toulouse ${ }^{7}$, les Néolithiques se sont installés sur la terrasse de $30 \mathrm{~m}$. de la Garonne, à Blagnac, Cugnaux, Saint-Martin-du-Touch et sur les limons sableux de la vallée de l'Hers. Un bon inventaire a été publié des stations néolithiques et de l'âge du Bronze dans le pays d'Apt $^{8}$. Des grottes funéraires, contemporaines du début de l'àge du Bronze, ont été fouillées à La Ripelle, sur le versant Sud du Mont-Combe, près de Toulon ${ }^{9}$ (perle de cuivre, pointes de flèches en silex).

(4) A. Cabrol et R. Robert, Fouilles d'abris aux Roches près La Ferte-Alais (Seine-et-Oise), ibid., XLII, 1945, p. 105-111.

(5) R. Pierron, Études de préhistoire cantalienne. Les civilisations lithiques du bassin d'Aurillac, dans Revue de la Haute-Auvergne, XXX, 1944, p. 194-211, 241-259. - Les haches néolithiques de Haute-Auvergne et les courants de culture préhistorique, ibid., XXXI, 1945, p. 10-29.

(6) G. Cordier, Contribution aux études de préhistoire de la Touraine. Découverte d'une station néolithique, atelier en forêt d'Amboise (Indre-et-Loire), dans B. S. P. F., XLII, 1945, p. 38-42.

(7) L. MÉroc, ibid., XLII, 1945, p. 124.

(8) Frédéric Lazard, Les environs d'Apt préhistorique. Etude sur le vallon de Buoux, le versant Nord du Lubéron et le plateau des Claparèdes, aux points de vue préhistorique et archéologique. Avignon, Rullières frères, 19.43, in- $8^{\circ}$.

(9) René Gérard, La station préhistorique de 
La sépulture de la tombe découverte dans la carrière Vergoignan, à la Frèche (Landes) ${ }^{1}$, avait été placée dans une anfractuosité naturelle, fermée par une murette en pierres sèches. Le squelette, qui porte des traces de combustion, était couché sur le côté gauche, les jambes repliées; a la hauteur de la poitrine, avait été installé un foyer, circonscrit par des pierres plates disposées en cercle, et dans une cavité contiguë on recueillit les restes d'un Ovidé. La sépulture de Wettolsheim a livré un curieux petit forel en silex pour le fraisage des grains de collier en coquillages ${ }^{2}$. L. Balsan poursuit activement ses recherches dans les grottes et les mégalithes aveyronnais ${ }^{3}$ et $\mathbf{R}$. Pouget décrit les dolmens et tumulus situés aux limites de ce département et de la Lozère. Aux environs de Montpellier, à Sainte-Croix-de-Quintillargues, au bois de Valène, et aux confins des communes de Mutelle, Cazevieille et Saint-Jean-de-Cuculle ${ }^{5}$, des dolmens et des stations ont été explorés qui appartiennent à l'Énéolithique. Le " tombeau "

la Ripelle, au Mont-Combe, près Toulon (Var), dans Bull. soc. de botan., géolog., entomolog. archéol. Var, no 55,1944, p. 12-15.

(1) Abbé Glony, Une sépulture néolithique dans les Landes, dans Bull. soc. préhist. Languedoc, no 2, 1945, p. 17-18.

(2) Du même, A la découverte des hommes préhistoriques. Explorations souterraines (1944), p. 131-145.

(3) I. Balsan, Fouilles archéologiques, 1943. Extr. des P.-V. soi. des lettr., sc. et aris Aveyron, 1944 ; du même, Fouilles archéologiques 19391940. Palethnologie aveyronnaise, extr., ibid., 1945.

(4) René Pouget, Dolmens et tumuli du Recoules de l'Hom, de Bombes, de Versel, d'Inos, dang Moun Clouquié. Bull. paroiss. de Novis (Aveyron), 1941-1942, p. 1943.

(5) $\mathrm{D}^{r}$ J. ARnal, La préhistoire à SainteCroix-de-Quintillargues (Hérault), dans B. S. P. F., XLI, 1944, p. 173-176; du même, Trois mégalithes inédits du bois de Valène, ibid., XLI, 1944, p. 112-116; 151-154. de Campet, à Benon ${ }^{6}$, près d'une source, sous tumulus, était fait d'un coffrage recouvert d'une carapace de pierres. Il semble appartenir à une phase ancienne du Néolithique. Le dolmen ruiné de La Bétoulle a été réoccupé à l'époque romaine $^{7}$. Quelques mégalithes ont été signalés en Touraine, dans la région de Crotelles-Villedômer et sur le plateau des Châtelliers, à l'Est du château d'Amboise $^{8}$. Un inventaire sommaire des dolmens et des cluseaux vient d'être présenté pour la vallée de l'Aussonne ${ }^{\boldsymbol{\theta}}$. Des tumulus et des enceintes ont été signalés, aux Pioliers, près de Randon ${ }^{10}$, des tombelles ovalaires au MenezHomne $^{11}$ et des dolmens dans le Jura ${ }^{12}$, ainsi qu'un puits à silex près d'Amboise ${ }^{13}$. Dans le parc de Pinterville, au fond d'un vallon, un ossuaire a été fouillé ${ }^{14}$, dans lequel les squelettes avaient été recouverts par des lits de

(6) J. Ferrier, La sépulture néolithique de Benon, commune de Saint-Laurent-Médoc (Gironde), extr. Bull. soc. linnéenne Bordeaux, 1943, 8 p., IV pl.; L.-D. Peyholles, B. S. $P$. F., XLII, 1945, p. 51-56.

(7) L. Bonnaud et J. Peirier, Le dolmen de la Bétoulle, dans Bull. soc. arch. et hist. du Limousin, LXXX, 1944, p. 367 et suiv.

(8) G. CoRdIER, Bull. trim. soc. arch. Touraine, XXIX, 1944, p. 59-60 ; p. 33-34, p. 36-37.

(9) Aubisse et du Chenon, Bull. soc. hist. et archéol. du Périgord, LXX, 1943, p. 257-264.

(10) H. Vertet, Vestiges anciens aux Pioliers, près Radan, dans Bull. soc. d'hist. et d'archéol. de Vichy, p. 263-266.

(11) D. Vourch, La préhistoire sur le MénezHom, dans Bull. soc. archéol. Finistère, LXVIII, 1941 (1943), p. 61-67.

(12) Marcel Baudor, Premier rapport sur la fouille du caveau sépulcral néolithique de Pinterville (Eure), dans Bull. soc. norm. ét. prêhist., XXXIV, 1944, p. 4-7.

(13) A. LePay, Les mégalithes du deparlement du Jura, dans B. S. P. F., XLII, 1945, p. 145147.

(14) G. Cordier, B. S. P. F., Xl.II, 1945, p. $148-149$. 
branchages, recouverts de pierres plates. $\mathrm{Au}$ sommet, on a recueilli un vase en terre noire, du type du Fort-Harrouard (Néolithique supérieur). Des mobiliers funéraires avaient été déposés avec les cadavres, pointes de javelot et poinçon en os, pendeloques et colliers, et près des corps d'enfants les ossements d'animaux familiers.

Une explication a été proposée pour les disques de pierre perforés : symboles religieux d'essence astronomique, originaires d'un centre eurasien ${ }^{1}$. M. Grünewald attire l'attention sur les modes de taille très variés des ateliers du GrandPressigny ${ }^{2}$.

La découverte la plus importante, relative aux arts du Néolithique et du premier âge des Métaux, est celle de peintures de style ibérique dans les grottes de la vallée du Caramy, au Sud de Tourves. Parmi les sujets représentés, on trouve de nombreuses figures anthropomorphes (idoles) et une chasse au renard (grotte Chuchy) ${ }^{3}$. A Tarascon-surAriège, un autre ensemble de ces peintures a été retrouvé, et dans les grottes du Grand-Père, des Églises Supérieures, de Sainte-Eulalie, du mont Saint-Béat, de Peyort, des représentations de guerriers, dont l'un tient une tête coupée, sont apparentées aux dessins de la grotte de Baldouin, près de Saint-Remyde-Provence. A l'occupation néolithique de la grotte de Saint-Jean-du-Roc, à Marcamps, se rattache une gravure anthropomorphe ${ }^{4}$. La même image de la

(1) A. Curtet, Contribution à l'étude de la signiflcation des disques perforés, B. S. P. F., XLI, 1945, p. 178-184.

(2) R. Grünewald, B. S. P. F., XliI, 1945, p. $138-140$.

(3) Glory, Savz Martinez, Neukirch, Les peintures rupestres de style ibérique dans la vallée du Caramy (Gard), dans B. S. P. F., XLI, 1944, p. 168-173.

(4) H. Lambert, Gravures pariétales de la déesse néolithique, gravée sur les dalles morbihannaises, se retrouve à l'hypogée de Coutinargue, sur le plateau du Castellet 5 . La gravure anthropomorphe du menhir de la Pierre-Quilhade, à Auriac, se rapproche vaguement de la statue-menhir, avec son poignard du Bronze I ${ }^{6}$. Les sculptures reconnues sur deux rochers à 1 kilomètre Sud-Sud-Est de Pont-Aven, au bord du sentier de la ferme du Bourgneuf, restent énigmatiques?

Peu de travaux ont pour objet l'âge du Bronze. Des inventaires ont été donnés de cette période pour le département de la Creuse $^{8}$ (quinze cachettes de fondeurs, des enceintes, des tombelles et des dolmens à incinération; aucun fond de cabane n'a encore été signalé) et pour la région libournaise ${ }^{9}$. Une nouvelle exploration a été conduite, au Trou-des-Morts, dans le massif de la Clappe $^{10}$, où $P h$. Héléna avait recueilli des céramiques du Bronze III. Le gisement a été pillé par les "chercheurs de trésors ", le souterrain-refuge de La Fer-

grotte Saint-Jean-du-Roc à Marcamp (Gironde), dans B. S. P. F., XLII, 1945, p. 48, 61 .

(5) Émile Bourlon, Nouvelles découvertes à l'hypogée de Coutinargue, plaleau du Castellet (commune de Fontvieille, Bouches-du-Rhône), dans B. A. C., 1941-42 (1944), p. 405-411.

(6) J. PÉrol, B. S. P. F., XLII, 1945, p. 28 29.

(7) E. Bolelli et R. P. Grot, Sculptures énigmatiques sur deux roches des environs de Pont-Aven (Finistère), dans B. S. P. F., XLI, 1944, p. 149-151.

(8) Dr G. Janicaud, Mélanges archéologiques (dixième série), dans Mém. soc. sc. nat. et archéol. de la Creuse, t. XXIX, 1944, extr. p. 1-23.

(9) Dः A. H. Bastris, L'áge du Bronze au Musée de Libourne, dans Rev. hist. et archéol. Libournais, no 43, 1944, p. 19-24.

(10) A. Glory, Etude d'une canine humaine de l'âge du Bronze. Trou-des-Morts (Aude), dans Bull. soc. préhist. Languedoc, no 2, 1945, p. 1415. 
rage, à Varages' ${ }^{1}$, se classe parmi les ossuaires du début du Bronze. La cachette de fondeur de Longueville ${ }^{2}$, au lieu-dit Monneron, entre un petit affluent de la Vézère et un vallon, contenait, dans un vase en terre ayant servi de creuset, des faucilles à bouton, des haches à talon, à tranchant non évasé, 1 pointe de lame à douille, 1 épée à languette, 1 poignard à rivets, des couteaux à lame courbe, des poinçons, 1 bélière, des épingles à collerettes, des anneaux-disques en tôle de bronze. L'ensemble peut être daté du Bronze III. Les faucilles sont arrivées dans le bassin parisien par l'intermédiaire de la civilisation palafittique de la Suisse et de la Savoie. Une petite cachette peut-être votive, de six haches à douille, a été trouvée dans la tourbe des marais du Trait, entre le chemin des Chantiers et la route du bac de la Meilleraye ${ }^{3}$. Une autre est signalée au passage à niveau de Courlandon, à la sortie d'Argentan ${ }^{4}$. En Limousin, une hache plate à rebords et deux à douilles ont été recueillies à Chàlus (Haute-Vienne) et à La Rivière, commune de Chassenon (Charente) ${ }^{\mathbf{5}}$.

A propos de l'emmanchement et des "marques de fondeurs" des faucilles, M. G. Gaudron ${ }^{6}$ remarque que le but de

(1) R. Corbeil, S. Royer, R. Girard, Souterrain-refuge et grotte de La Ferrage. Station inédite à Varages (Var), dans B. S. P. F., XLI, 1944, p. 91-103.

(2) Henri Lamarre, La cachelle de fondeur de Longueville (Seine-et-Marne), dans Rev. archeol., 1945, I, p. 98-115.

(3) J. Bailly, Note sur la découverte de haches en bronze, faite au Trait, le 20 juin 1942, dans Bull. soc. norm. ét. préhist., XXXIV, 1944, extrait.

(4) Bull. soc. nat. antiq. Fr., 1942, p. 157.

(5) Bull. soc. archéol. et hist. Limousin, LXXX, 1944, p. 102-103, 118.

(6) G. Gaudron, Faucilles à boulon. Leur emmanchement, leurs marques de fondeur, dans B. S. P. F., XLI, 1944, p. 159-164. la faucille est de couper une poignée d'épis ou de tiges, tenue de la main gauche, sans que l'autre main soit obligée à un mouvement de torsion trop pénible : le tranchant doit donc présenter par rapport au manche une obliquité comprise entre 45 et $90^{\circ}$. Quant aux prétendues "marques de fondeur", elles correspondent à des précautions prises par le fondeur pour nourrir de métal la lame à sa base, et par le taillandier pour renforcer un point faible, au moyen de nervures, et assurer une meilleure fixation de l'outil dans le manche de bois.

\section{Les Ages du Fer}

Comment concilier les données de l'archéologie et les textes des auteurs anciens qui laisseraient supposer l'existence d'un domaine marseillais, dont Avignon et Cavaillon auraient fait partie? Dans une étude récente, ce problème n'a pas été abordé, l'auteur n'ayant utilisé que les sources littéraires et n'ayant pas tenu compte de la ceinture d'oppida indigènes qui isole Marseille de l'intérieur du pays?.

Dans ma précédente chronique, j'ai déjà attiré l'attention sur l'importance de la stratigraphie du Cayla de Mailhac, pour la chronologie des stations préromaines du Languedoc méditerranéen. De nouvelles précisions sont apportées par M. Henri Martin-Granel ${ }^{8}$. Une suite de forteresses pré-romaines, installées sur les contreforts méridionaux des Cévennes et sur les collines des Corbières, jalonnent comme une frontière autour

(7) J. Brunel, Etienne de Byzance et le domaine marseillais, dans R. E. A., XLVII, 1945 , p. 122-133.

(8) Henri Martin-Granel, Les fouilles de l'oppidum du Cayla, à Mailhac (Aude), dans Gallia, III, 1944, p. 1-14. 
de la région Narbonne-Béziers. La voie naturelle Océan-Méditerranée est barrée par le seuil de Naurouze, le Carcassis et le Minervois, chaque coupure importante étant fermée par un verrou. L'importance du Cayla décroit par rapport au développement d'Ensérune. Il semble qu'après l'invasion des Volques, l'ancienne frontière de l'Ouest narbonnais perdit, avec son caractère ethnique, sa raison d'être. C'est alors que les marchés, comme Ensérune, redoublent d'activité.

La pénétration des Celtes, en Bretagne, au ve siècle avant J.-C., eût pour conséquence la mise en culture du territoire, fait qui avait été déjà constaté en Champagne. Des noms de lieu, Méreuil, Les Beauces, gardent le souvenir de ces défrichements. Le pays était alors couvert de bois, mais avec de vastes clairières, où s'abritent les exploitations agricoles ${ }^{1}$.

Les fouilles de Mellecey ${ }^{2}$ au bord de la voie d'Agrippa, reliant Chalon-surSaòne à Autun, ont donné l'occasion d'étudier une station routière d'origine protohistorique. A un établissement néolithique peu important succède une station de La T'ène III, qui ne disparaitra qu'au Moyen âge, lorsqu'une hôtellerie s'installera à sa place. Il apparait que le site, dépourvu d'eau et dénué de toute protection naturelle, a été uniquement choisi parce qu'il était placé à un carrefour. Le matériel celtique recueilli offre de grandes analogies avec celui du MontBeuvray. Des scories de fer et quelques culots de bronze marquent l'emplace-

(1) Guy Sociliet, Le peuplement et la mise en valeur d'une commune d'Ille-el-Vilaine de la préhistoire au Moyen-Age. Laillé, dans Bull. et mém. soc. archéol. Ille-et-Vilaine, LXVII, 1944, p. 39-97.

(2) L. Armaxd-Caldiat, Fouilles châlonnaises, dans Ann. Bourgogne, XVI, 1944, p. $62-63$. ment d'un atelier de fondeur. Mellecey est un grile d'élape entre le grand oppidum de Bibracte et son port d'embarquement de Chalon.

La continuation des fouilles de Gergovie $^{3}$ a confirmé l'existence, déjà reconnue, de trois enceintes et de trois couches archéologiques successives, correspondant à une occupation hallstattienne, puis à un habitat des deux derniers siècles avant notre ère, caractérisés l'un par des poteries campaniennes, l'autre par des céramiques italiotes. A signaler pour la petite histoire de Gergovie, les "souvenirs" de Marcellin Boudet, sur Napoléon III et Gr.rgovie ${ }^{4}$. L'occupation du plateau de Corent (Puyde-Dôme),à 16 kilomètres de ClermontFerrand, près de la route d'Issoire, a pu commencer avec l'époque de Hallstatt. Un habitat gallo-romain recouvre des huttes gauloises. De nombreux tessons ont été ramassés, dont certains, à décor peint, appartiennent aux ateliers lédosiens de La Tène III ${ }^{5}$.

Le Vivarais ${ }^{6}$ apparait comme un pays de refuge et de lien entre la montagne et les territoires rhodano-méridionaux. Les Celtes, en position d'atlente dans les Cévennes, ont défriché la forêt d'yeuses et de chênes rubescents et, sans doute, introduit le châtaignier au cours du second âge du Fer, continuant l'œuvre des pasteurs néolithiques et de l'àge du Bronze qui avaient conquis, pour le passage de leurs troupeaux de moutons, les crêtes alpines.

Des caps barrés, au Combalou et à

(3) A. Grenier, CRAI, 1944, p. 395-396.

(4) L'Auiergne, lahiers d'études régionales, no 110 , p. 5-19.

(5) Robert Terrisse, Bull. soc. nat. antiq. Fr., 1942 , p. 108-142.

(6) M. Lovis, Civilisation cévenole et vocation spirituelle du Vivarais, dans Revue du Vivarais, 1942, p. $69-71$. 
Saint-Georges-de-Luzançon, des cimetières hallstattiens, à Floyrac et sur le plateau du Larzac, précisent les conditions de l'installation des (ieltes en Aveyron ${ }^{1}$. Dans la Creuse, on a relevé l'existence de plus d'une centaine de tumulus, certains d'origine hallstattienne, réutilisés pour des tombes gallo-romaines, et ayant finalement joué le rôle de mottes féodales ${ }^{2}$. Dans la région antique de Troyes, un vase portant le graffite $\Delta$ śxuou a été recueilli rue Surgale ${ }^{3}$. Une tombe de cavalier, avec ses armes et ses objets de parure, a été découverte dans le cimetière gaulois de Nonant-le-Pin ${ }^{4}$.

Une excellente étude sur la diffusion d'un type d'armes, le poignard à antennes, comme critère de l'extension d'un groupe humain, a été donnée par G. Fabre ${ }^{5}$. Ces poignards, caractéristiques de la civilisation posthallstattienne qui s'étend de la Castille à la Garonne, ont une origine celtique. Mais ils constituent des catégories qui ne sont pas contemporaines et n'ont pas la même aire de répartition. En Aquitaine, on rencontre seulement les types à antennes redressées $\dot{a}$ angle droit, contemporains des $v^{e}-$ Ive siècles. En Languedoc, ils sont accompagnés d'une poterie peinte caractéristique. La civilisation posthallstattienne de l'Aquitaine traduit l'arrivée de formes industrielles dont l'évolution s'est faite en Espagne et en Languedoc. Elle correspond à une poussée ibérique entre

(1) L. Batsan, Foutlles archéologiques, 1943, dans P.-V. soc. des lett., sc. et arls Aveyron, extrait.

(2) Dr G. Janicaud, op. cit., p. 20-23.

(3) Chandon de Briailles, Bull. soc. nat. antiq. Fr., 1942, p. 159.

(4) Du Mesnil Du Buisson, ibid., 1942, p. 158.

(5) G. FABre, Les poignards it aniennes de l'âge du Fer tronvés dans le sud-Ouest de la France, dans Bull. suc. archéol. Midi France, V, 1943, p. 56-66.
Garonne et Pyrénées. Les épées de La Tène II languedociennes représentent l'entrée des Volques dans la région toulousaine, alors qu'en Aquitaine persiste un vieux fonds hallstattien, avec seulement quelques infiltrations celtiques (tombe de femme de Mazerolles, tumulus d'Aubagnac. T. II). On ne retrouve des Celtes que sur les confins, dans le pays de Buch, à Agen, Toulouse, Saint-Bertrandde-Comminges, là où un demi-siècle plus tôt s'étaient arrêtés les porteurs des grandes épées de bronze et de fer. Alors qu'en Aquitaine, la première période de La Tène n'est pas représentée, on constate les traces évoluées d'un vieux fonds celtique du Bronze et du premier Fer. Il est à remarquer que précisément ces éléments sont ceux-là mêmes que les Ibères ont emprunté aux Hallstattiens. Le glaive des mercenaires d'Hannibal n'a pas été importé de Gaule en Ibérie, et ce n'est pas l'épée des Celtes de La Tène II qu'ont adoptée les Romains, mais un dérivé du poignard hallstattien à antennes. Aquitains, comme Ibères, ont reçu des (ieltcs du vie siècle, leurs armes, leurs bijoux et parfois aussi leur céramique. Seuls bons métallurgistes, dans un pays où le minerai de fer est abondant, ils ont conservé les types en les perfectionnant. Les deux peuples ont entretenu des rapports pacifiques qu'attestent la numismatique, la toponymie et l'onomastique et, en les confondant, les auteurs anciens n'ont retenu que les éléments posthallstattiens, partout ailleurs disparus.

Alors que l'invasion celtique du vi ${ }^{\theta}$ siècle a laissé des traces en Languedoc et en Castille, elle n'est pas représentée en Aquitaine. Aux temps de la descente des Ibères en Languedoc, on constate une identité de civilisation entre Aquitaine et Castille. L'invasion des ('eltes de La Tène II a laissé de côté l'Aquitaine. 
Les limites de cette aire de civilisation correspondent à celles de la numismatique ibérique et de la séparation des langues d'oc et d'oïl. Elle s'étend sur les plateaux, régions d'élevage du mouton et du cheval. Originaire des champs d'urnes de l'Europe centrale, cette culture descend jusqu'en Catalogne ; une autre vague pénètre dans la haute vallée de la Garonne, alors que les (Celtes porteurs de la grande épée de fer ne peuvent entrer en Aquitaine. Une étude plus serrée de l'évolution du poignard à antennes permet de rétablir les conditions dans lesquelles s'est fait le peuplement de l'Aquitaine pendant l'âge du Fer. Les Celtes du vire siècle ne sont pas passés d'Aquitaine en Espagne, puisqu'ils n'ont laissé aucune trace d'un séjour dans cette province. D'autre part, il est impossible d'attribuer cette culture aux Ibères, mais l'aire de dispersion des poignards à antennes de la deuxième phase ( $\left(\mathrm{v}^{-I v^{e}} \mathrm{~s}\right.$.) déborde l'Aquitaine au Nord de la Garonne. Le mouvement semble correspondre à un reflux d'éléments celtiques, stationnés en Languedoc et en Espagne, sous la poussée d'envahisseurs ibériques entre Garonne et Aquitaine. Il n'est donc pas possible d'attribuer à une occupation ibérique les noms d'Eliberre, Eluza, Calagurris, Iluro, Oloro, Tolosa, Carcaso. Cette toponymie remonte bien plutôt aux temps des navigations ibériques de l'âge du Bronze.

La traduction exacte du mot gaesum était restée imprécise. Selon un essai d'explication nouveau ${ }^{1}$, le terme celtique ne se bornerait pas à désigner une arme, mais aussi des outils agricoles, comme l'aiguillon armé d'une pointe, la guise $\mathrm{du}$ Baujolais, du Charollais.

La hache en fer, trouvée dans le lit

(1) J. Descrois, Qu'est devenu le Gaesum gaulois? dans REA, XLVII, 1945, p. 153-155. de la Marne, à Bisseuil², a conservé son manche, fait d'un morceau de bois, sectionné au-dessus d'un nœud; l'amorce de la branche forme la crosse qui s'insère dans la douille, on obtient ainsi un manche fait d'une seule pièce. Bien que restant énigmatiques, il semble que, par comparaison avec les bracelets de jambes du pays Sao, on puisse reconnaitre dans les ornements penannulaires de section triangulaire des pièces votives représentant des anneaux de chevilles ${ }^{3}$.

Une obole massaliote a été recueillie dans un ruisseau à Saint-Remy-deProvence ${ }^{4}$; dans la carrière de la Pérelle, près de Champ-Cars, en Saint-Jacquesde-la-Lande ${ }^{5}$, un trésor de monnaies gauloises des Redons était renfermé dans un vase en terre-cuite ; une monnaie des Baiocasses a été découverte dans la région d'Argentan ${ }^{6}$.

Parmi les sculptures récemment publiées, on signalera un griffon de bronze (Halls'att II $B$ ), provenant du lit de la Loire, entre Les Ponts-de-Cé et Saint-Gennes, objet d'importation ionienne d'assez grandes dimensions ${ }^{7}$; une statue d'homme, en pierre, aux paupières closes, un torques autour du cou, la main droite, ramenée sur la poitrine, tenant un poignard de ses six doigts repliés, trouvée à Pauvrelay (Indreet-Loire $)^{8}$. Une bonne reproduction a été

(2) Abbé Favret, Rev. archéol., 1945, 2, p. 138-139.

(3) R. Lantifr, Bull. soc. nat. antiq. Fr., 1942, p. 86-90.

(4) H. Bailland, Rev. numis., 5" sér., VIII, 1944-1945, p. 191.

(5) Bull. et mém. soc. archéol. Ille-et-Vilaine, L.XVI, 1941, p. Xxir.

(6) Du Mesnil DU Buisson, Bull. soc. nat. antiq. Fr., 1942, p. 157.

(7) DP M. GRUET, Rev. archéol., 1945, 2, p. 123-127.

(8) Baron H. Auvray, B. S. $P$. $F$, XliI, 1945, p. 18-19; Chassaing, ibid., p. 102-104. 
donnée d'une petite image de guerrier celtique, conservée au musée de Strasbourg' .

Deux très importants ouvrages de P. Jacobsthal et Fernand Benoît ${ }^{2}$, traitent de l'art celtique. Alors que dans le premier, il s'agit principalement de l'art ornemental et décoratif, le second apporte une très intéressante contribution à l'histoire de la sculpture celtique en Provence et dans le Languedoc méditerranéen.

\section{V. Époque Gallo-Romaine}

1. Les cités. - P.-M. Duval a conduit des fouilles sur le site de Cemenelum $(\text { Cimiez) })^{3}$ : un amphithéâtre, parmi les plus petits connus, des aqueducs amenant l'eau du versant Nord des hauteurs, des demeures privées ont été mises au jour. La ville joua, depuis le règne de Néron, le rôle d'une sorte de capitale en miniature jusqu'à la veille de l'organisation de Dioclétien, époque à laquelle elle est rattachée au diocèse de Vienne. Cimiez eut à souffrir de la rivalité de Nice, sa voisine, mieux partagée avec son port. Lorsque les troubles amenèrent la déchéance des relations commerciales par la voie de terre, Cimiez perdit toute importance. A Orange ${ }^{4}$, sous les masures situées à l'Est du théâtre,

(1) A. RgFF, Gaulois et Germains sur la terre $d^{\prime}$ Alsace, Strasbourg, Oberlin, 1945 , in- $8^{\circ}, 20$ p., 7 pl.

(2) P. Jacobsthal, Early cellic art, Oxford, Clarendon Press, $1944 ; 1$ vol. de texte, 1 vol. de pl. in-4\%. - F. Benolt, L'art primitif méditerranéen de la vallée du Rhône. La sculplure. Paris, Éd. d'art et d'histoire, 1945, in-40. Voir : R. LANTiER, L'art cellique, dans Journal des Savants, 1945, p. 67-77.

(3) Ch. Samaran, CRAI, 1945, p. 167-168. L. Cappati, Cimiez dans l'histoire, Armanac Nissart, 1943, p. 9-96.

(4) J. Formigt, Bull. soc. nat. antiq. Fr., 1942, p. $105-106$. de "petites maisons ", élégamment décorées de mosaïques et de peintures ont été mises au jour. Au sujet de l'ouvrage du chanoine J. Sautel sur Vaison, M. Albert Grenier ${ }^{5}$ présente quelques pertinentes observations : de la prédominance des marques de potiers de La Graufesenque ne pourrait-on conclure que l'existence de la cité ne commence vraiment qu'avec le règne de Tibère? La construction en forme de $\mathrm{T}$ du prétoire est-elle bien un autel? Elle paraît bien grande. Au reste, si des inscriptions mentionnent un praefectus à Vaison, on ignore s'il s'agit d'un magistrat municipal ou d'un délégué de l'administration romaine.

Deux rapports établis par la Commission des fouilles de Saint-Bertrand-deComminges ${ }^{6}$ font connaitre les résultats des recherches effectuées, de 1933 à 1938, au forum et dans les quartiers voisins de la ville basse. L'angle septentrional du forum est occupé par de grands thermes publics, construits sur les substructions d'un balnéaire contemporain du principat d'Augusle, lors des travaux de restauration entrepris dans ce quartier au début du règne de Trajan. Le monument est en rapports directs avec le péribole du temple, prolongement des portiques du forum, dont il n'est séparé, au Sud-Est, que par un mur mitoyen. On est ainsi en présence d'un grand ensemble monumental, comprenant place publique, thermes et théâtre, de même appareil et de même époque, dispositions déjà reconnues à l'autel du Confluent à Lyon, et aillcurs, et qui viendraient à l'appui de l'hypothèse du

(5) A. Grenier, REA, Xl.VI, 1944, p. 371.

(6) Rapport sur les fouilles de Saint-Bertrandde-Comminges (Lugdunum Convenarum) de 1933 à 1938, dans Mém. soc. archéol. Midi Fr., XX, 1943, p. 205-248; XXI, 1945, p. 17-87. 
rôle joué par Lugdunum Convenarum, en tant que capitale fédérale des tribus pyrénéennes, entre Garonne et Aquitaine. Au Nord-Est des thermes, dans le quartier dit "Les Vignettes", sous les ruines d'insulae, occupées par des habitations privées, des maisons plus anciennes, en bois ou en pisé, représentent les vestiges de la plus ancienne agglomération (72 av. J.-C.). Aux temps des Antonins, sur ces emplacements s'étendent des quartiers d'habitation, en bordure du decumanus et desservis par des rues se coupant à angle droit. De grunds thermes publics occupent toute la superficie de l'insula 2 ; agrandis et embellis au début du III $^{\mathrm{e}}$ siècle, ils furent reconstruits sous le règne de Constantin et disparurent avec la cité, au ve siècle, lors de l'invasion des Vandales. A l'extrémité opposée de la ville basse, au Nord et à l'Est de la basilique chrétienne, des sondages ont révélé l'existence de nouveaux quartiers d'habitation et, au Nord et à l'Est, celle d'un grand bâtiment public. Dans la ville haute, au jardin des Olivétains (ancien évêché médiéval), une piscine appartient à un grand édifice et, sous la nouvelle salle du musée, s'étend la crypte d'une église mérovingienne. A Bazeret, autour du sanctuaire, s'était formée une petite agglomération gallo-romaine.

S'appuyant sur les conditions géologiques et physiques du site, M. Broëns ${ }^{1}$ combat l'hypothèse d'une cité à VieilleToulouse qui aurait été à l'origine de Toulouse romaine. Il n'y a pas eu d'oppidum tectosage en cet endroit. L'existence, sur l'emplacement même de Toulouse, d'un castellum, élevé vers 120 avant l'ère par le consul Cépion, est plus vraisemblable. Quatre ruelles, dans la ville moderne, déterminent assez bien

(1) M. BROËNS, CRAI, 1944, p. 341-342. ce retranchement. La cité romaine était très étendue. On connaît d'assez nombreux restes de son enceinte de briques, mais peu de monuments : un temple sous l'église de la Daurade; dans le lit de la Garonne, un théâtre et un amphithéâtre; à 4 kilomètres au delà du fleuve, des ruines d'aqueducs sur la route de Cugnoux. Une étude du réseau antique des égouts apporterait des précisions sur la topographie.

C'est au jeudi 11 octobre, en 43 avant J.-C., qu'il convient de fixer la date de la fondation de Lyon ${ }^{2}$.

Les travaux d'urbanisme et de défense passive ont apporté quelques précisions à la topographie antique de Limoges ${ }^{3}$ : traces d'habitations place de la République sur l'emplacement occupé plus tard par l'abbaye de Saint-Martial ; dans la région méridionale de Périgueux ${ }^{4}$, peut-être les restes du portique d'une villa ; à Orléans ${ }^{5}$, rues Chappon, SaintFlou, Charles-Sanglier, Coulon, SainteCatherine, au cloître Saint-Aignan, tuiles, poteries et monnaies.

A Vichy ${ }^{6}$, des travaux effectués par

(2) A. Audis, La fondation de Lyon, dans Ass. lyonn. recherches archéol., 1945, p. 4-8.

(3) E. Vincent, Dans les tranchées-abris de Limoges, dans Bull. soc. archéol, el hist. Limousin, LXXXI, 1945, p. 187-199.

(4) P. Barrière, Découverles récentes à Périgueux, dans Gallia, III, 1944, p. 245-25I.

(5) Bull. soc. archéol. et hist. Orléanais, XXIV, 1942 (1944), p. 265-266, 270-271, 272, 238, 241, $242,248,249,258$.

(6) $\mathrm{D}^{\mathrm{r}}$ A. Mortet, Captage gallo-romain de la source de l'Hôpilal, dans La Presse médicale, no 30,14 août 19.13 , p. 445-446; Dr L. Chabrol, Béton de briques et béton romain, dans Bull. soc. d'hist. et d'archéol. de Vichy et de ses environs, 1944 , p. 224-239 ; Dr A. Mortet, lichy romain. Puits romains de Chomel, extrait de Vichy médical, mars 1945, 9 p.; du même, La cité des Aquis Calidis, extrait, ibid., mai 1945, 11 p.; du même, Adduction d'eau de source aux Aquis Calidis, ibid., 1944. 
la Compagnie fermière des Eaux à la source de l'Hôpital, ont amené la découverte du captage romain. La source jaillissait à gros bouillons d'un puits maçonné, autour duquel avait été coulé un épais massif de béton, interdisant toute déviation latérale des eaux. A la partie supérieure, une margelle, formant vasque, entourait le puits et, sur l'un des côtés, un orifice circulaire assurait l'écoulement de l'eau. La vasque portait un revêtement de marbre, et, dans l'antiquité, le dallage sur lequel elle reposait était au niveau du sol. Des restes importants de piscines ont été reconnus dans le voisinage, sur la place du monument aux morts. La présence d'installations hydrothérapiques, au Hall des Sources, et la découverte de tasses en terre-cuite servant à l'absorption des eaux, ainsi que la présence d'un important radier de béton à l'Est du puits Chomel, engagent à reconnaître l'emplacement de l'établissement de bains galloromains, autour des griffons actuels des sources Chomel et de la Grande-Grille. Au Sud-Est, la source Lucas, au-dessus de laquelle s'élevait peut-être un frigidarium, était également comprise dans les bains. Quelques indications ont été apportées à l'étude des canalisations : un tuyau de plomb, portant les noms des empereurs Septime-Sévère et Caracalla, a probablement une origine locale. La présence d'un important établissement thermal eut pour conséquence le développement du centre urbain, dans lequel on pénétrait par les ponts du Séchon et de l'Allier, et un certain nombre de voies dont les tracés ont été en partie suivis, et que bordaient, à la périphérie, les cimetières de La Ville-aux-Juifs et du quartier du Callou. Certains quartiers paraissent avoir été réservés à une catégorie déterminée d'artisans : les potiers, nombreux à Vichy, au champ
Capelet, près de la gare, à la Croix des Renards, rue Desbret; les forgerons et les bronziers, place de la Liberté. Des édifices religieux ont été signalés rue Callou et rue Victoria et une petite officine de faux monnayeurs, rue du MaréchalFoch. Pour assurer l'alimentation en eau potable, les eaux de la source de FontFiolent furent amenées par un double aqueduc, l'un se dirigeant directement sur Vichy, qu'il atteignait en plein centre, sur l'emplacement de la gare du chemin de fer; l'autre suivait la vallée du Séchon et pénétrait dans la ville par le quartier de la Ville-aux-Juifs. Tous les deux aboutissaient au bassin collecteur, découvert dans le jardin Maussang, rue Nationale. Un système d'égouts assurait l'évacuation des eaux usées.

Faut-il localiser Genabum à Orléans ou a Gien? Sans prendre nettement parti, après un exposé détaillé et une critique des sources littéraires et archéologiques, G. Chevallier ${ }^{1}$ laisse deviner qu'il penche pour Orléans.

Les curieux de l'histoire des fouilles d'Alésia se reporteront au mémoire de J. Toutain ${ }^{2}$. Ils y trouveront également une description générale des ruines. Les campagnes de 1944 et 1945 ont porté sur le cimetière Saint-Père, les parcelles cadastrales 633,634 , et dans la région située à l'Est du forum. Elles ont apporté des précisions à la connaissance des routes et chemins passant à travers la cité et précisé la topographie générale

(1) Guy Chevaldien, Genabum, Orléans ou Gien? Synthèse d'hisloire et d'archéologie régionales. Orléans, M. Houzé, 1944, in-8 ${ }^{\circ}$.

(2) J. Toutain, Quarante années de fouilles à Alésia, dans Mémorial des Etudes latines, 1943, p. 628-640; du même, Les fouilles exécutées da Alise-Sainte-Reine (Côte-d'Or), en 1943, par la Société des sciences historiques et naturelles de Semur, dans Gallia, III, 1944, p. 121-140; du même, Ann. Bourgogne, 17, 1945, p. 225. 
du site. Un examen attentif du cadastre permet de restituer le plan des cités romaines. C'est le cas pour Senlis ${ }^{1}$, dont on ne connait que les arènes. L'important cimetière mérovingien ne peut s'expliquer que par l'existence d'une nombreuse population gauloise, puis gallo-romaine. Anderitum (Andrézy) aurait été entre Paris et Rouen, l'étape principale de la navigation fluviale et le point d'attache de la flottille assurant la protection du trafic ${ }^{2}$. Et c'est parmi la population de la localité et des environs, bateliers, mariniers, nautes de la Seine, que les empereurs auraient recruté les équipages. Un habitat romain a été reconnu dans la cour du Petit Séminaire d'Amiens ${ }^{3}$.

Où placer le portus Aepatiaci? A Isques, à une lieue de Boulogne, siège d'une garnison nervienne 4 .

Le nom de Nevers dans l'antiquité était primitivement Nibernum, dérivé sous forme neutre de celui de la Nièvre, les habitants étaient les Niberni, nom tardivement fixé au locatif Nibernis, de là Nevers ${ }^{5}$. De nouvelles hypothèses sont proposées ${ }^{6}$ pour expliquer le nom de Beaune, rendant peut-être mieux compte de l'association Beleno, Belina, Belenein.

(1) G. Matherat, Bull. soc. nat. antiq. Fr., 1942, p. 197-212.

(2) J. Toutain, Le problème d'Andrézy-surSeine. Andrezy dans l'antiquité, dans B. A. C., 1941-2, p. 505-518.

(3) Abbé Ch. Martin, Bull. soc. antiq. Picardie, 1944, p. 259-260.

(4) Jules Vannerus, "Portus Aepaliaci, dans R. E. A., XLVI, 1944, p. 299-317; A. Grenier, CRAI, 1944, p. 372-386.

(5) E. Thévenot, Le nom de Nevers, sa valeur semantique et son évolution, dans Bull. soc. nivern. lettr., sc. et arts, XXI, 1941, p. 420-429.

(6) P. LEBEL, Le nom de Beaune, nouvelles notes, dans Ann. Bourgogne, 16, 1944, p. 103108.
Dans la construction des villes romaines, les mêmes gestes se sont reproduits aux mêmes endroits, pour des raisons de commodité ou de dispositions naturelles des lieux : c'est ainsi que les bains, les marchés, les temples occupent bien souvent des emplacements identiques. Les mêmes phénomènes se constatent presque partout : transfert au point fort, du capitole à la forteresse des invasions, puis médiévale. De là une certaine uniformité dans le plan des cités gallo-romaines : lots initiaux généralement assez petits (20 à 30 mètres en moyenne), réunis aux dépens des rues pour l'assiette des grands monuments; un tracé général établi rigoureusement, en se basant sur le point de l'horizon où le soleil se levait le jour de la fondation; un périmètre qui se plie au relief du sol; un débordement des remparts, contemporains de la fondation, par les constructions qui s'étendent et donnent naissance à d'énormes faubourgs?

2. Les monuments. - A la scène du théâtre d'Orange, certaines maladresses dans l'exécution témoignent de la part prise dans la construction par les vétérans de la II légion. Les éléments de la décoration, les marbres ont été, eux, apportés directement de la carrière, prêts à être mis en place. Les sculptures, malheureusement en pièces, sont également importées. A la Porte Royale, audessus du chambranle, courait une frise de danseuses et de danseurs et, de part et d'autre de l'ouverture, dans des niches, se dressaient des statues, dont une de Vénus. Un entablement de quatre grandes colonnes cannelées, en marbre blanc, flanquait la porte, en avant de quatre pilastres. L'ensemble était couronné par

(7) J. Formige, Bull. soc. nat. antiq. Fr., 1942, p. 283-289. - P. Grimal, Rev. de philologie, XIX, 1943, p. 163-174. 
une frise de Centaures et de Centauresses. Au-dessus de la corniche du grand ordre, un large espace rectangulaire était ménagé pour l'inscription de la dédicace que surmontait une grande niche centrale, abritant une statue colossale d'Auguste, le torse protégé par une cuirasse, un prisonnier barbare agenouillé à sa droite. En arrière du grand mur du postscenium s'étendaient des portiques, servant à la fois de foyer et de promenoir, recouverts de charpentes légères, décorées de peintures et d'appliques de bronze. Le sol était dallé de marbres ou de mosaïques. Qrange est le seul théâtre où ce dispositif a pu être étudié ${ }^{1}$.

Une reconstitution de l'orchestre du théâtre d'Arles a pu être esquissée ${ }^{2}$ : l'autel aux Cygnes, conservé au musée, était la thymélé sur laquelle on plaçait aux jours de fêtes une statuette de bronze de Bacchus. Deux autres autels, portant également un décor augustéen, appartenaient à cet ensemble.

L'existence du théâtre de Civeaux, soupconnée par Maximin Deloche, est mainlenant certaine ${ }^{3}$. Il n'en est pas de même pour l'amphithéâtre supposé de Chalon-sur-Saône ${ }^{4}$, sur lequel on ne possède aucun renseignement précis.

Poursuivant ses recherches sur les sanctuaires gallo-romains à tombe de héros, A. Grenier ${ }^{5}$ ajoute à la liste établie le temple des Cars, à Saint-Merd-les-

(1) J. Formige, dans Bull. soc. nal. antiq. Fir. 1941, p. 45-50, 172-180.

(2) Du même, L'autel aux Cygnes d'Arles et la thỵmélé dans les théâtres gréco-romains, dans Rev. archéol., 1944, 1, p. 21-34.

(3) Eygux, Bull. soc. antiq. Ouest, $3^{\circ}$ sér., XIII, 1944, p. 347.

(4) L. Armand-Calliat, L'amphithédire supposé de Chalon-sur-Saône, dans Mérn. soc. d'hist. et d'archéol. Chalon, XXXI, 1945, p. 57-177.

(5) A. Grenier, Sanctuaires gallo-romains et tombe du héros, complement à la communication du 6 août 1943, dans $C R A I, 1944$, p. 221-229. -
Oussines, dans la région de Millevaches. C'est un slibaleion, qui se dresse au sommet du lioilon du grand theâtre de Vienne (Isère), à l'exemple du temple qui, à Rome même, couronnait, en quelque sorte, le théâtre de Pompée ${ }^{6}$. De ce monument provient vraisemblablement la décoration sculpturale dionysiaque en partie retrouvée : un Silène, adossé jadis à un mur, comme les Papposilènes du stibadeion délien, les chapiteaux aux serpents dressés de part et d'autre d'un trépied. On possède enfin quelques précisions sur les découvertes faites au temple du Moulin du $F \hat{a}^{7}$, dont les substructions et les abords immédiats ont été reconnus : construit sur plan circulaire, au centre d'un podium également circulaire, il possède une cella, ouverte au Sud-Est et précédée par un parvis rectangulaire. L'enceinte, repérée en deux points en direction du théâtre, comprend deux murs parallèles, distants de 7 mètres, ce qui permet de supposer l'existence d'une galerie. Derrière la cella s'ouvrent deux puits : tombes de héros? Près de Saint-Gaudens, à SaintPlancard, la découverte de stèles dédiées à Mars Sutugius laisse présumer l'existence d'un lieu de culte sur l'emplacement de l'église dans les murs de laquelle les monuments ont été remployés ${ }^{8}$.

De brèves descriptions ont été données des aqueducs des Clausonnes, alimentant Antibes ${ }^{9}$ et de l'aqueduc amenant à

J. Formigt, Les fouilles de Sanxay, dans Gallia, III, 1944, p. 43-97.

(6) Ch. Picard, Un type méconnu de lieusaint dionysiaque: le stibadeion, dans CRAI, 1944, p. 152-153.

(7) L. Basalo, Le temple de Moulin $d u F \hat{a}, a$ Barzan, près Talmont-sur-Gironde (CharenteMaritime), dans Gallia, III, 1944, p. 141-164. Résumé par A. G[RENier], p. 164-165.

(8) A. Rey, CRAI, 1945, p. 519.

(9) J. Formigte, Bull. soc. nat. antig. Fir., 1942 , p. 227. 
Tours les eaux des fontaines de Cléré, dont le tracé a été reconnu sur les territoires d'Athée, de Véretz, et dans le parc du château d'Azay-sur-Cher, où la conduite franchit un petit vallon au moyen de trois arcades ${ }^{1}$.

La monographie que R. Dauvergne ${ }^{2}$ consacre aux thermes des FontainesSalées est un modèle du genre. Ce n'est pas une simple description des ruines, mais une étude approfondie de la vie du site, des raisons qui ont conduit les hommes à s'y installer. Les fouilles sont replacées dans leur réalité physique et nous disposons d'une exacte reconstruction du paysage gallo-romain, bien différent de celui qui se déroule aujourd'hui sous les yeux du visiteur. Le sort de ce petit coin de terre a été, jusqu'à la Révolution, toujours réglé en fonction du sel, et c'est le sel qui est à l'origine des modifications profondes subies par le site au cours de cette histoire.

3. Occupation territoriale et voies romaines. - La Forma orbis romani s'est enrichie d'une nouvelle carte, celle du département de l'Aveyron ${ }^{3}$ et, sous le titre ambigu - je ne sache pas que l'on ait jamais discuté à ce sujet de l'utilité des travailleurs provinciaux, É. Thevenot, pour le pays éduen, L. Armand-Calliat pour le Chalonnais, R. Louis pour l'Yonne, G. Drioux pour le pays de Langres, G. Grémaud pour le

(1) H. Auvray, Bull. trim. soc. archéol. Touraine, XXIX, 1944, p. 30.

(2) Robert Dauvergne, Sources minérales, thermes gallo-romains et occupation du sol aux Fontaines-Salees, commune de Saint-Père-sousVézelay. Paris, 1944 , in- $8^{\circ}$, 129 p., 8 fig. 6 plans, VII pl.

(3) Forma orbis romani. Fasc. IX, Carte archéologique de la Gaule romaine, dressée sous la direction de M. Adrien Blanchet. Carte et texte complet du département de l'Aveyron, préparés par M. Émile Bonnet, complétès et terminés par le directeur, Paris, 1944.
Dijonnais ${ }^{4}$ apportent de très utiles compléments à l'esquisse de la feuille de Lyon que j'avais donnée pour la Tabula imperii romani. Les résultats d'une enquête menée sur les noms de lieu de l'arrondissement de Chalon-sur-Saône ${ }^{5}$ dépassent les limites modestes où l'auteur a voulu les maintenir. Le travail est riche en hypothèses et en suggestions qui demandent à être vérifiées par des recherches sur le terrain. Toutefois, il n'est pas douteux qu'à un nom de lieu est souvent attaché un fait de folklore : baune, balma, touchent à la préhistoire; pierre désigne souvent un menhir; folletière est à rapprocher de Pierre-aux-Fées; motle, tartre, tertse indiquent un tumulus; châtelet, châtelot, une enceinte. L'archéologie confirme ces toponymes. Un curieux exemple est donné par le nom de Cercot, forme abrégée de sarcophagus : des sarcophages ont été découverts à Cercot même. La carte dressée par l'abbé Lestocquoy ${ }^{6}$, pour les découvertes gallo-romaines du Pas-de-Calais, fait connaitre un peuplement important dans un cercle d'une vingtaine de kilomètres de rayon, dont le centre serait à Arras, le long de la route de Brunehaud et dans le Boulonnais. La région LensBéthune-Arras est riche en découvertes mérovingiennes, mais le problème du peuplement, aux $\mathrm{v}^{\mathrm{e}}$ et $\mathrm{vi}^{\mathrm{e}}$ siècles, est loin d'être résolu.

Après les justes et sévères critiques de J. Soyer, on croyait en avoir fini avec les erreurs doctoralement proclamées sur le désert de la Beauce à l'époque romaine.

(4) Annales de Bourgogne, XVI, 1944, p. 1528 (cartes).

(5) L. Armand-Calinat, Pour la carte archéologique du Chalonnais. Indications toponymiques tirées des lieux et des notes de folklore, extrait des Mém. de la soc. d'hist. el d'archéol. de Chalonsur-Saône, XXX, 1943, p. 137-196.

(6) Reıue du Nord, XXVII, 1944, p. 163-164. 
Mais M. Dauzat ${ }^{1}$ a relevé le gant, oubliant que nombre de domaines baucerons, comme le remarque A. Grenier, ont été attribués par les rois mérovingiens, carolingiens, voire capétiens, comme fiefs à leurs fidèles. C'est alors qu'ils ont pris les noms de leurs nouveaux possesseurs. De telles erreurs ne font que déconsidérer la toponymie qui doit s'incliner devant les enseignements apportés par les fouilles archéologiques. Dans la succession des circonscriptions administratives sur un même coin de terre, il faut tenir compte autant de la terre que de l'élément humain. Toutes ne sont pas devenues des pagi ${ }^{2}$. Ceux que nous connaissons appartiennent aux temps mérovingiens; de ceux contemporains de l'époque romaine, on possède quelques noms, et leurs limites restent incertaines. On possède maintenant une bonne enquête sur les pagi de la Domnonée au $\mathrm{IX}^{\mathrm{e}}$ siècle $^{3}$.

Deux études ${ }^{4}$ précisent les conditions de l'occupation dans les environs d'AliseSainte-Reine depuis les temps préhistoriques; des camps et des enceintes occupent les hauteurs voisines, des villages gaulois s'échelonnent sur le chemin de Bacarat à Grésigny, et pour l'époque romaine on connait plus d'une douzaine

(1) A. Dauzat, R. E. A., XLVI, 1944, p. 319.

(2) Abbé Chaume, Une question. La succession des circonscriplions sur un même coin de terre, dans Ann. de Bourgogne, XVI, 1944, p. 162-174, 229-240.

(3) R. Coufron, Les pagi de la Domnonée au $I X^{\theta}$ siècle, d'après les hagiographes bretons, dans Mém. soc. hist. el archéol. Brelagne, XLIV, 1944, extrait 24 p., 4 cartes.

(4) E. Fornerot, Carte d'Alise et de ses environs, d'apres les noles sur Alise et ses environs de Victor Pernet, dans Soc. sc. hist. et nat. de Semur-en-Auxois, c. r., 1942, p. 21-22; J. Toutain, Les vestiges antiques autour du Mont-Auxois (canton de Venarey), dans id., 1943, p. 7-9, 15-16. de sites correspondant à des domaines, formant la grande banlieue d'Alésia. Le territoire et les limites de la civitas Leucorum concordent avec la ligne de démarcation de l'ancien diocèse de Toul, comme l'a établi pertinemment, dans une excellente étude, M. Toussaint ${ }^{5}$. En Auvergne, la "plate " comprise entre la petite vallée de la Beautourne et celle du ruisseau de Vinlat, a été habitée jusqu'aux temps chrétiens. Son abandon a été provoqué par le déboisement qui a contraint les occupants à chercher un refuge sur les versants abrités ${ }^{6}$. En Bourgogne, l'exploration du territoire de Mellecey? a donné d'intéressants résultats, une station routière à laquelle succède une Maison-Dieu, une étude du cadastre et du terrain de la commune de Laillé $^{8}$ a fait connaitre l'existence, à l'époque romaine, de plusieurs grands domaines fonciers, cellules économiquement autonomes, à la fois agricoles et industrielles (exploitation du minerai de fer). Le paysage breton n'est pas sans rappeler alors les "champagnes" du bassin parisien, du Nord et de l'Est de la France. Les Gallo-Romains n'ont pas connu le bocage avec le cloisonnement de ses haies. La carte des noms de lieux ne représente que l'habitat concentré du Bas-Empire et n'a conservé aucune trace des nombreux écarts, disparus à la suite des invasions du IIr ${ }^{\mathrm{e}}$ siècle de notre ère. Un même phénomène de concentration de l'habitat reparait en Bretagne

(5) Maurice Toussant, Le territoire et les limites de la "civitas Leucorum", dans B. A. C., 1941-2, p. 413-428.

(b) M. Vazeilles, Bull. hist. et scientif. Auvergne, 1945, extrait. 4 p.

(7) L. A.-Calliat, Ann. Bourgogne, XVI, 1944, p. 62-63; Mém. soc. d'hist. et d'archéol. Chalon-sur-Saône, XXIX, 1940, p. 76-79; Gallia, 3, 1944, p. 25-41.

(8) Guy Souillet, op. cit. 
avec les incursions des Normands et la guerre de Cent Ans.

Il est difficile d'évaluer la population des villes gallo-romaines et l'on sera reconnaissant à $\mathbf{M}$. F. Lot ${ }^{1}$ des précieuses indications qu'il réunit à ce sujet.

De nouvelles découvertes ont été faites à Trinquetaille ${ }^{2}$ sur l'emplacement du cimetière moderne; à la PetiteGarenne, près de La Seyne ${ }^{3}$, un hypocauste a été mis au jour. Sur la route d'Avignon, à Cavaillon ${ }^{4}$, au quartier des Vergers, des puits avaient été forés depuis l'époque du Hallstatt jusqu'au II ${ }^{\mathbf{e}}$ siècle après J.-C. pour l'alimentation en eau des agglomérations antiques. Dans la Loire, le petit village de Piney ${ }^{5}$ est riche en antiquités : enceintes faisant partie du système défensif du fleuve, digue-pont romain et pont antique à Saint-Rambert-sur-Loire. En Aveyron ${ }^{6}$, des établissements gallo-romains ont été reconnus au Monal, aux Crottes, à La Glène, à Girman, et une monographie a été consacrée à Millau ${ }^{7}$, dont les deux premiers chapitres traitent de la préhistoire et de l'époque gallo-romaine. On possède un inventaire pour les découvertes faites dans la région de Sévérac,

(1) Ferdinand Lot, Hecherches sur la population et la superficie des cités remontant à la période gallo-romaine. Bibl. Ec. Haut. Et., sc. hist. et philolog., fasc. 287. Mâcon, 1945 (1 re partie).

(2) F. Benort, Recherches nouvelles ḋ Trinquetaille, dans Gallia, III, 1944, p. 251-257.

(3) Bull. soc. botan., génlog., entomolog. et archéol. Var, no 55, 1944-5, p. 2.

(4) A. Dumoulin, Les puits antiques de Cavaillon (Vaucluse), extrait Bull. soc. ét. sc. nat. Vaucluse, 1943, nos 3-4, p. 22.

(5) J. Renaud, Note sur les antiquites de Piney, dans Bull. Diana, XXIX, nos 2-3, 1945, p. 81-86; du même, Les pilotis du vieux pont de Saint-Rambert-sur-Loire, dans id., p. 98-99.

(6) L. Balsan, Fouilles archéol., 1939-40, 1943.

(7) Jules Artiéres, Millau à travers les ages. encore peu explorée ${ }^{8}$. A Chassenon ${ }^{9}$, un nouveau puits a été fouillé qui a donné une petite réglette graduée en marbre, et à La Boissière ${ }^{10}$, on a dégagé une piscine circulaire, avec accès par un double escalier. En Périgord, la villa de la Cornedie $^{11}$, à Piégut-Pluviers, est à la fois un centre agricole et industriel (ferrières). En Touraine, dans le jardin du presbytère de Ports-sur-Vienne ${ }^{12}$, des chapiteaux et des fûts de colonne marquent l'emplacement d'un important édifice gallo-romain. On connait encore mal les antiquités d'Azay-le-Rideau ${ }^{13}$ : tombe de la Rémonière, dont le château s'élève sur les ruines d'une villa ou d'un grand édifice. La villa d'Anrosay ${ }^{14}$, située au voisinage d'une source, a été réoccupée après les invasions et des tombeaux ont été installés dans ses ruines, avec des pierres de démolition. Au champ de l'Os, près de Vitteaux (Côte-d'Or), une villa a été reconnue en $1867^{15}$.

Le Nord de la France, à l'écart des grands courants commerciaux, occupe une situation défavorable au point de vue des communications. Aucune grande voie, si favorable au transport des marchandises, ne le traverse. Cet isolement explique le conservatisme des formes, en particulier pour la céramique : il est trop difficile d'y amener les produits

(8) Albenque, Journal Aveyron, 4-12-43.

(9) Dr P. Masfrand, Bull. soc. hist. et archéol. Limousin, LXXXI, 1945, p. 172-176.

(10) Ibid., LXXX, 1944, p. 123.

(11) P. Barrière, L'établissement de la Cornedie, dans Gallia, 3, 1944, p. 240, 245.

(12) Baron H. Auvray, Bull. trim. soc. archéol. Touraine, XXIX, 1944, p. 30.

(13) Du même, Azay-le-Rideau gallo-romain, ibid., XXIX, 1944, p. 43-53.

(14) P. Lebel, Vestiges archéol. découverts a Anrosay (Haute-Marne), dans Rev. archeol., 1945,2 , p. 88-92.

(15) De Montuamont, Doc. sc. hist. el nat. Semur-en-Auxois, c. r., 1942, p. 20. 
des officines arvernes. L'administration romaine, en établissant le réseau routier, a eu surtout pour préoccupation de faciliter les transports de troupes et la transmission des ordres, puis les communications avec l'ile de Bretagne et les pays rhénans ${ }^{1}$. La voie Regordane, reliant la Provence à l'Auvergne, suit en de nombreux points de son tracé le cours des rivières. On peut se demander si en Gaule, comme en Terre-Sainte, ce nom n'est pas en rapport avec le chemin d'eau, le guide, le conducteur du voyageur $^{2}$. La région de $\mathrm{Mazan}^{3}$, traversée par de nombreux ruisseaux descendant du Ventoux ou de la chaine de Vaucluse, occupe dans le Comtat une situation presque centrale, au débouché du passage mettant les vallées du Rhône et de la Durance, en communication par d'anciens chemins avec Digne et Sisteron, Vaison et Cavaillon. On doit signaler des études sur les voies romaines de Vichy ${ }^{4}$, rectifiant le tracé de la route Périgueux-Saintes, aux environs de Chancelade $^{5}$, les chemins savoyards ${ }^{6}$, les rapports des chemins ruraux et de la centuriation romaine dans la plaine châlonnaise ${ }^{7}$, la voie

(1) Roger Dron, Les voies romaines du Nord de la France, étudiees sur la carte, Publications de la Soc. de géogr. Lille, 1944-5, p. 535.

(2) A. Blanchet, Hypothèse à propos de la voie Regordane, dans CRAI, 1945, p. 458-463.

(3) Léon Ciarrias, Les vieux chemins du terroir de Mazan (Vaucluse), dans B. A. C., t. V, 13 novembre 1944 , p. vI-vir.

(4) H. Waller, Les voies anciennes des Aquis Calidis, dans Bull. soc. d'hist. et d'archéol. de Vichy, 1944, p. 100-115.

(5) Mercier, Bull. soc. hist. et archéol. Périgord, LXXII, p. 96.

(6) Émile Vuarnet, Mém. el doc. publ. par Acad. Chablaisienne, XLVII, 1943, p. Xv.

(7) André Délíage, Le réseau des chemins ruraux dans la plaine chalonnaise et la centuriation romaine, dans Mém. soc. d'hist. et d'archéol. Chalon-sur-Saône, XXIX, 1940, p. 146-151. romaine de Naix à Langres 8 , la station routière de Bolar ${ }^{9}$, les traces du cadastre romain en Bretagne ${ }^{10}$, la route de Paris à Rouen dans son passage à travers le Parisis et le Vexin français ${ }^{11}$, la chaussée Brunehaut de Bavay à Reims ${ }^{12}$.

4. Les sépultures. - Des fouilles seraient à entreprendre pour déterminer le caractère du monument, dit " La Chèvre d'or", dressé à Biot (AlpesMaritimes), sur une hauteur dominant la vallée. Ést-ce un tombeau, semblable à celui de La Penne, près de Marseille ${ }^{13}$ ? Une tombe sous tuile a été découverte à Port-Issal ${ }^{14}$ et les scouts ont fouillé un cimetière bouleversé des $\mathrm{II}^{\mathrm{e}}-\mathrm{IIII}^{\mathrm{e}}$ siècles, près de la chapelle de Fontibus, à l'angle Nord-Ouest du cimetière de Salinelles, commune de Sommières ${ }^{15}$.

Une nouvelle étude de J.-J. Hatt ${ }^{16}$

(8) G. Davillet, La voie romaine de Naix (Nasium) da Langres (Andemalunnum), dans $B A C, 1941-2$, p. 441-450.

(9) E. Thévenot, Conjectures sur la station antique de Bolar à Nuits-Saint-Georges, dans Ann. Bourgogne, 1944, p. 242-247.

(10) A. Meynier, Traces de cadastres romains en Armorique, dans CRAI, 1944, p. 413-422; du mème, ('hamps et chemins en Bretagne, dans Conférences universitaires de Bretagne, 1942-3, p. 161-178.

(11) J. Toutain, Note sur la voie romaine de Lutece à Rotomagus (Rouen) dans le Parisis et en Vexin trançais, de Paris à Saint-Clair-surEpte, dans BAC, p. v., 12 juin 194.t, p. v-xvir. (12) M. Henault. La voie romaine de Bavay à Reims (dite chaussée Brunehaut). Essai de reconstitution de son tracé anlique. Lees slations. Les colonnes milliaires, dans B. A. (.., 1941-2, p. 451-469.

(13) J. Formige, Bull. soc. nat. antiq. Fr., 1942, p. 225-227.

(14) Bull. soc. botan. géolog., entomolog. et archéol. Var, no 55, 1944, 5, p. 4.

(15) Clan archéologique des Chênes Verls. Fontibus, nécropole gallo-romaine. Les Éclaireurs de France, s. d., in- $8^{\circ}, 15$ p.

(16) J.-J. Hatr, Les monuments funéraires gallo-romains du Comminges et du Couserans, dans Ann. Midi, LIV-LV, 1945, p. 169-254. 
apporte des éclaircissements sur les conditions dans lesquelles se développèrent les coutumes de l'art funéraire dans le Comminges et le Couserans. L'épigraphie permet de mettre un peu d'ordre dans la chrolonogie de ces mouvements : l'emploi de la formule $h(i c)$ $s$ (itus) $e(s t)$ est contemporaine du I $^{\text {er }}$ siècle de notre ère; la dédicace au datif parait à la fin du Ier et au début du II $^{\mathbf{e}}$ siècle et, à partir du milieu du II $^{\mathbf{e}}$ siècle, on ne relève plus que la formule $D$ (is) M(anibus). Les monuments, en forme d'auges, représentent une variété locale de la stèle-maison gallo-romaine et continuent la tradition indigène des caissons funéraires de l'âge du Bronze. Ils témoignent aussi de la longue survivance de certaines croyances celtiques sur l'autre monde, situé dans les régions sidérales, sur les mythes de la transmigration des âmes, dans lesquels une divinité de caractère astral et funéraire, Epona gallo-romaine ou Rhiannon celtique, joue un rôle prépondérant. On retrouve volontiers dans les représentations des défunts se versant à boire le souvenir d'un ancien rite hallstattien. $\mathrm{Au}$ point de vue social, ces curieux monuments précisent l'importance prise, aux II $^{\mathbf{e}}$ et III $^{\mathrm{e}}$ siècles, par les communautés de caractère à la fois professionnel, cultuel et funéraire dans les groupes indigènes de la Gaule. L'art funéraire local, purement romain aux origines, se libère, au cours du second siècle, de ces influences : motifs et symboles funéraires ne relèvent plus désormais que d'une inspiration empruntée aux croyances celtiques. Il est intéressant de mettre en rapport ce renouveau des modes indigènes avec l'expansion de la romanisation qui pénètre dans les contrées les moins accessibles du pays. C'est aussi le temps où les productions en série des marbriers gallo-romains se répandent de plus en plus dans toutes les classes sociales. Mais pour suivre le goût du public elles tendent de plus en plus à prendre une physionomie locale. Stèles, cippes et auges se couvrent de motifs nouveaux, signes astraux, instruments d'artisans, et de très anciens thèmes décoratifs, héritiers du passé celtique.

Une sépulture à inhumation d'époque romaine a été trouvée dans le cimetière néolithique du Verdier ${ }^{1}$. Contrairement à la thèse soutenue par $F$. Benoît, le mausolée de Lanuéjouls (Lozère) doit être rapporté au I $^{\mathrm{e}}$ siècle, au temps d'Hadrien; les images des Victoires rappellent autant celles des arcs de triomphe provençaux que celles des sarcophages du III $^{\mathbf{e}}$ siècle. Enfin l'excellence de l'appareil interdit de reconnaitre une construction de basse époque ${ }^{2}$. Des cimetières ont été fouillés à Riesse (Lozère) ${ }^{3}$ avec poteries de Banassac, à la Maison-Blanche ${ }^{4}$, Iv ${ }^{\theta}-v^{e}$ siècles, à Issoire $^{5}$, II $^{\mathrm{e}}$ siècle, sur le territoire de la commune de Giat, lieu dit "Chez Rozet ", milieu du II $^{\mathrm{e}}$ siècle $^{6}$, près de Planartige et au Champ du Puy de la Faux (Cor-

(1) R. Terrisse, Sépulture à inhumation au cimetière néolithique du Verdier (Tarn-etGaronne), dans Bull. soc. nat. antiq. Fr., 1942, p. 31-32.

(2) J. Formigé, ibid., 1942, p. 78-82.

(3) Dr Ch. Morel, Gallia, III, 1940, p. 133240.

(4) E. Desforges et P. F. Fournier, La nécropole de la Maison-Blanche (commune de Pardines), dans Revue d'Auvergne, 59, 1945, extr. 23 p.

(5) J.-J. HATt, Tombes à incinérations décou. vertes à Issoire, dans Revue d'Auvergne, LVIII, 1944, extr. 15 p.

(6) J. Charbonneau, Découverte d'une sépulture gallo-romaine d'incinération près ChezRozet (commune de Guat), dans Bull. hist. et archéol. Auvergne, LXIV, 1944, extr. 
rèze $)^{1}$, au Chalard ${ }^{2}$. Un inventaire a été établi pour les sépultures antiques de la Vienne $^{3}$. La tombe de La Geneytouse, à La Gorce, a fourni un mobilier funéraire comprenant des bijoux d'or, bague, anneau et éléments de collier (III ${ }^{\mathrm{e}}$ siècle? $)^{4}$. Au cimetière d'Allichamps ${ }^{5}$, des stèles funéraires à personnages ont été utilisées pour la confection d'un sarcophage. Il ne s'agit pas de la découverte d'un cimetière de l'âge du Fer, à Lanslevilard $^{6}$, mais de tombes romaines des premiers siècles de notre ère.

5. Arts el métiers. - Ce que l'art roman doit à l'art celtique a été précisé par P. Francastel ${ }^{7}$ : loi du cadre; thèmes gallo-romains passés dans le répertoire des imagiers médiévaux ; réapparition des traditions artistiques indigènes.

Le bas-relief de la face méridionale du mausolée de Saint-Remy-de-Provence ${ }^{8}$ représente deux scènes distinctes : à droite, la chasse de Méléagre; à gauche, le massacre des fils de Niobé, au cours d'une chasse dans les forêts de Cithéron. Par les caractères de souplesse du dessin,

(1) Bull. soc. archéol. el hist. Limousin, LXXX, 1944, p. 85. - Dr Janicaud, Mém. soc. sc. nat. et archéol. Creuse, XXIX, 1944, p. 29-31.

(2) Ibid., LXXXI, 1945, p. 70.

(3) F. Det.AGE, Sépullures antiques de la Vienne, dans Bull. soc. émul Bourbonnais, 1942, extrait.

(4) Du même, Nọ́tes d'archéol. g.-r. II, Sépulture de la Gorce, dans Bull. soc. hist. ei archéol. Limousin, 1945, p. 219-226.

(5) Bull. soc. antiq. Centre, 1941-2 (1945), p. XI.

(6) J. Bellet, Notes sur la préhistoire de Lanslevilard (Savoie), Belley, 1932.

(7) P. Francastel, Sculpture gallo-romaine et sculpture romane, dans Rev. archéol., 1944, 2, p. 134-142.

(8) Fr. Chamoux, Sur un bas-relief du mausolée de Saint-Remy, dans CRAI, 1945, p. 177183. l'audace des raccourcis, le goût de l'action violente et du pathétique, la composition relève, par l'intermédiaire des "cahiers de modèles", des grandes $œ u v r e s$ de la peinture grecque du Ive siècle avant notre ère.

La borne de pêche, sur laquelle on voulait jadis reconnaitre un seigneur et un moine, représente, d'après le dessin inexact qui subsiste, deux époux sur une stèle funéraire. La pièce était en place, aux $\mathrm{XvI}^{\mathrm{e}}$ et $\mathrm{XvII}^{\mathrm{e}}$ siècles, aux environs de Saint-Germain-du-Plain (Saône-etLoire $)^{9}$. Le nettoyage de la statue de Champs, lors de son entrée au musée de Guéret, a permis d'identifier le monument, non pas avec une image de Mercure, mais avec une déesse-mère tenant un enfant $^{10}$. Malgré une nouvelle étude ${ }^{11}$, les reliefs de l'autel de Verneuil-Moutiers restent toujours inexpliqués. Un important complément est apporté à la liste des sculptures gallo-romaines du pays éduen ${ }^{12}$. La tête d'homme, trouvée à Alésia, entre les ruines d'un temple et le monument à triple abside, appartient probablement au personnage masculin d'un groupe du dieu et de la déesse assis $^{13}$. Le masque en bronze du musée de Senlis se rattache à la série de pièces semblables,découvertes en forêt de Compiègne et à Garancières-en-Beauce ${ }^{14}$. Le démontage, à l'atelier du musée des Antiquités nationales, du groupe d'Epona avec le poulain découvert au hameau de La Sarrazine, en 1860 , a démontré que

(9) L. Armand-Calliat, $B A C$, p.-v., 17 lévrier 1944, p. v-x.

(10) Dr J. Janicaud, Mém. soc. sc. nat. el archéol. Creuse, 1944, p. 24-27.

(11) F. Delage, Notes d'archéol. g.-r., II, L'Autel de Verneuil-Moutiers, dans Bull. soc. hist. el archéol. Limousin, 1945, p. 213-218.

(12) Ann. Bourgogne, 17, 1945, p. 68-69.

(13) J. Toutain, $B A C$, p.-v., 12 mars 1945.

(14) R. Lantier, Bull. soc. nat. antiq. Fr., 1944, p. 215-217. 
le socle est une adjonction moderne, dont la faiblesse a nécessité l'établissement d'un coffrage de bois pour supporter le poids des figurines. Malgré la fente en tire-lire, le socle n'a jamais pu être utilisé, comme on l'avait supposé, comme tronc d'offrandes ${ }^{1}$. Une statuette du dieu au maillet, de la collection Ch. Fabre, à La Gagère (Puy-de-Dôme), provenant de Lezoux, témoigne des affinités existant entre Mercure et le dieu au maillet, qui est figuré ici avec des chaussures à talonnières ailées, allusion au rôle psychopompe de Sucellus ${ }^{2}$. A l'occasion du legs fait par M. Alfred Lacroix, secrétaire perpétuel de l'Académie des Sciences, au Musée des Antiquités nationales, de la collection réunie à Mâcon par son grand-père Tony Lacroix, une description des statuettes de bronze a été donnée ${ }^{3}$. On remarque spécialement une figurine de dieu chasseur, tenant un lièvre par les pattes de derrière, sujet déjà connu par la statuaire de pierre, mais encore inconnu dans les séries de petits bronzes figurés. Le musée de Poitiers vient de s'enrichir d'un magnifique bronze grec, trouvé dans le département de la Vienne, représentant un athlète vainqueur se couronnant de la main droite et de l'autre main tenant une bandelette. Dans cette œuvre, peut-être une copie de l'époque d'Hadrien se manifeste une influence polyclétéenne ${ }^{4}$.

On doit au chanoine Drioux ${ }^{5}$ un inventaire des mosaïques et des peintures murales découvertes à Langres, et à G. Matherat la publication de deux instruments de mesure découverts à

(1) Du même, ibid., 1942, p. 123-125.

(2) Du même, ibid., 1942, p. 160-163.

(3) Du même, ibid., 1942, p. 166-171, 181-189, 190-195.

(4) Guillon, CRAI, 1945, p. 227-229.

(5) Abbé Drioux, B.A.C., 1941-2, p. 429-440.
Senlis $^{6}$. H. Rolland ${ }^{7}$ a donné un relevé des inscriptions antiques de Saint-Remyde-Provence, revision et complément du c. I. $L$.

Des ateliers de potiers importants existaient sur le territoire du département de l'Aveyron, à La Graufesenque, Banassac, Saint-Pierre-du-Trépied et à Cahors. Des tuiliers travaillaient à Camboulet ${ }^{8}$. Parmi les marques de potiers, trouvées dans la Haute-Vienne, celles des officines de La Graufesenque sont plus nombreuses que les estampilles arvernes. Peu de marques sont encore connues en Corrèze ${ }^{9}$. Aux premiers temps de l'Empire, l'industrie de la terre-cuite était florissante à Vichy ${ }^{10}$, dont les ateliers avaient une spécialité de glaçure plombifère.

Ce ne sont pas des céramiques de La Tène, mais des vaisselles communes, fabriquées à Rome au cours de la seconde moitié du I ${ }^{\text {er }}$ siècle avant J.-G., qui ont servi de modèles aux potiers "belges " ${ }^{11}$.

On retirera grand profit, pour la datation de la poterie d'usage domestique, des précisions données par J.-J. Hatt ${ }^{12}$ sur les tessons trouvés au fond d'un puits, à Clermont-Ferrand, sur l'emplacement des nouvelles facultés. Les fragments de

(6) G. Matherat, Bull. soc. nat. antiq. Fr., 1942, p. $59-78,281-283$.

(7) H. Rolland, Gallia, III, 1944, p. 167-223.

(8) A. VIré, Potiers et tuiliers gallo-romains du Quercy et du Rouergue, dans Bull. soc. ét. litt. scientif. archéol. Lot, 1943, p. 204-217.

(9) F. Delage, Inventaire des marques de potiers gallo-romains trouvées à Limoges et dans la Haute-Vienne, dans B. A. C., 1941-2, p. 471497 ; du même, Marques de potiers gallo-romains recueillies dans la Corrèze, dans B. A. C., 1941-2, p. 499-504.

(10) Dr A. Morlet, Vichy gallo-romain. Prototypes céramiques; - Enochoés; - Les tasses de Vichy;-Lampes; extraits de Vichy médical, 1945. (11) OxE, Trierer Zeitschft., 1941-2, p. 92-104. (12) J.-J. Натт, Bull. hist. el archéol. Auvergne, LXIV, 1944, extrait. 
sigilléc sont tous antérieurs au règne des Flaviens.

On connaissait déjà l'existence de six fours, à l'atelier de potiers de 'Thuisy ${ }^{1}$ (Marne), en activité à l'époque augustéenne. Un septième plus récent possède un foyer séparé de la chambre de cuisson et, à chaque extrémité, une aire de travail, alors que, dans les six autres, le foyer est en mème temps chambre de cuisson. Celle-ci était recouverte d'une voûte en branchages, supportant une carapace de terre durcie par la mise à feu de cette armature. La fournée se composait de ollie ovoüdes classiques de lype belge, en terre fine (début $\mathrm{i}^{\mathrm{er}}$ siècle) et d'assiettes, dont quelques-unes à engobe noir. La plupart des estampilles relevées dans le sous-sol de Lyon appartiennent à La Graufesenque et à Montans; aux ${ }_{11}{ }^{e}-I_{11}{ }^{e}$ siècles, on trouve des vases provenant des officines de la vallée du Rhône ${ }^{2}$.

Le Rouergue méridional est riche en exploitations minières ${ }^{3}$ et, en Corrèze ${ }^{4}$, des ateliers ont été signalés dans le canton de Juillac. Il semble que la dispersion des lingots de plomb chalonnais puisse être mise en rapports avec les événements de 197 ap. J.-C. Des trains de bateaux ou des dépôts de lingots auraient été pillés alors, mais la victoire de Septime-Sévère sur Albinus aurait empêché les pillards d'emporter leur butin au delà d'une vingtaine de kilomètres de Chalon-sur-Saône, où ces pièces ont été retrouvées ${ }^{5}$.

(1) M. BRy, L'alelier céramique de Thuisy (Marne), dans Gallia, 111, 1944, p. 229-232.

(2) P. Wunleumier, Ass. lyonn. de rech. archéol., 1944, p. 9-16.

(3) J. Rouquette, P.-v. soc. des lett. el des arts Aveyron, 21 octobre 1943.

(4) Marius Vazeilles, Mines de fer a SaintSalve (Correze), dans Bull. soc. scienlif., hist. et archeol. Corrèze, 67, 1945, p. 132-134.

(5) L. Armand-Calliat, les lingots de plumb
6. La Religion. - Il est regrettable qu'une étude qui apporte un tel ensemble de documents sur les Dioscures en Gaule et en Germanie ${ }^{6}$ soit entièrement faussée par un détestable chauvinisme, et relève d'une doctrine où les idées raciales, arbitrairement introduites dans la protohistoire, témoignent d'une absence totale de sens historique. Il est faux de poser en principe que le culte des Dioscures, dans le Nord et l'Est de la Gaule, représente des influences germaniques. Si le caractère des Alces est incontestablement germanique, les Dioscures ont été apportés au delà du Rhin par les Celtes et, s'il est nécessaire de chercher des rapports avec l'extérieur, c'est du côté du monde gréco-romain qu'il faut regarder. Ce qu'il y a, dans cette histoire, non pas de germanique, mais de commun aux Celtes et aux Germains aux temps de la communauté indo-européenne, ce sont les images de chevaux, de cerfs et d'élans - l'alces de César - matériel indistinctement fourni aux deux groupes alors indivis. L'histoire n'enseigne pas la primauté des Germains mais la rapide ascension des Celtes, bien antérieurement libérés des liens communautaires. Comment alors admettre une action des premiers sur les seconds, et cela aux environs de l'ère chrétienne, alors que le contact est rompu? Il y a des mythes dont il faut se débarrasser, surtout en histoire.

La représentation de la légende troyenne sur un vase, découvert à Cavaillon et originaire d'Arezzo, ne montre pas seulement la popularité de ce thème à l'époque augustéenne, mais encore le

chalonnais et les événements de 197, dans Ann. Bourgogne, 1944, p. 240-241.

(6) E. KRüGER, Die gallischen und germanischen Dioskuren. Die Martes Divanno et Dinomagetimarus und die Alces, dans Trierer Zeitschft., 16-17, 1941-1942, p. 1-66. 
caractère religieux qui s'attache à la conquête des Gaules par l'association de la bordure de casques gaulois à l'une des scènes les plus célèbres de la guerre de Troie : Cassandre réfugié près du palladium, à demi-dévêtue par Ajax qui l'entraine, et renversant la statue d'Athèna qu'elle tenait embrassée. La pièce appartient à une série de propagande pour l'exportation'.

7. Numismatique. - A Glanum (SaintRemy-de-Provence), un pilier avec base, fronton et patère sculptée au sommet, porte une inscription indiquant qu'un légionnaire en congé libérable a dédié à Mars un aureus et l'a déposé dans un autel placé à l'entrée du temple. Or, vers le milieu du texte gravé, on constate la présence d'une petite cavité rectangulaire où la pièce de monnaie aurait pu être fixée ${ }^{2}$.

On connait peu la circulation monétaire dans les provinces romaines du Midi, au I $^{\text {er }}$ siècle avant l'ère. La cachette de Bessan apporte quelques éclaircissements $^{3}$. Elle contenait une forte proportion de quinaires dont l'émission fut reprise de 49 à 44 par César, peut-être à cause de la faveur dont jouissait en Gaule cette monnaie. Le trésor du Cannet $(\text { Var })^{4}$ fut probablement enfoui au Sud de la voie Fréjus-Brignoles, par crainte de troubles à la mort de Septime-Sévère. La cachette de Tôtes (Seine-Inférieure) contenait 1.600 petits bronzes saucés

(1) F. Benoit, La légende troyenne sur un vase de Cavaillon, dans Bull. soc. ét. des sc. nat. de Vaucluse, 1944, p. 23-24.

(2) H. Rolland, P.-v. soc. fr. numism., 1945, p. XLVIII-L.

(3) P. Le Gentilhomme, La trouvaille de Bessan (Hérault), dans Rev. num., 1943, p. 145148.

(4) H. Rolland, Un trésor de monnaies romaines découvert au Canet (Var), dans id., 1943, p. 149-159. d'argent aux effigies des empereurs du III $^{\mathrm{e}}$ siècle ${ }^{5}$. Des monnaies de Gallien à Aurélien ont été recueillies à Soulièvre, près d'Airvault (Deux-Sèvres) ${ }^{6}$. Le dépôt de monnaies, enfoui au col du Chat, vers le milieu du Ive siècle, de même que les trésors de Réotier (Hautes-Alpes), de Saint-Vincent-de-Mercuze (Isère) et de Bredannaz (Haute-Savoie) correspond aux invasions que Julien dut repousser en Gaule. En 357, les Lètes dévastent la région lyonnaise?

Des aurei de Dioclétien et de Maximien Hercule auraient été frappés dans un atelier gaulois, probablement à Meaux ${ }^{8}$, base de Maximien Hercule et de Constance Chlore lors des préparatifs de la campagne contre Carausius. Mais cette hypothèse résout-elle le problème?

Dès les Antonins et les Sévères, on constate une tendance à la thésaurisation des espèces de bon poids. Au III $^{\mathrm{e}}$ siècle, la circulation de l'or se restreint, de là la présence des bijoux et des barres de métal précieux dans les trésors, et la décentralisation de la frappe des monnaies d'or est la conséquence des incessants déplacements de l'empereur (guerres civiles et étrangères $\left.{ }^{9}\right)$.

Aux $v^{e}$-vi ${ }^{e}$ siècles, la frappe de l'or est activement poussée chez les Barbares. Mais les ateliers officiels sont désertés pour les officines privées, qui procurent le numéraire aux cités et aux campagnes. Le droit de monnaie est exercé par les églises. Il arrive un moment où, devant la carence de la menue monnaie, on met en circulation des pièces gauloises ou

(5) O. Costa de Beauregard, Bull. soc. normande ét. préhist., XXXIV, 1944, p. 17-18.

(6) Rev. numis., 1945, 5, p. 189.

(7) P. Wullleumier, Rev. numis., 1944-5, p. 193-194.

(8) P. Le Gentilhomme, Bull. soc. nat. antiq. Fr., 1942, p. 83-86.

(9) Du même, Rev. numis., 1943, p. 11-43. 
romaines et des fragments métalliques, qui sont alors plus communs que les rares pièces d'argent ou de bronze frappées par les Barbares ${ }^{1}$.

\section{Temps Cinitiens et Múrovingiens}

Le livre d'A. Loyer ${ }^{2}$ fait revivre une période angoissante de l'histoire de la Gaule, en un temps où une société de beaux esprits, oublieux des bouleversements politiques et sociaux, assiste impuissante à la fin d'un monde, s'amusant à polir des acrostiches indolents.

Sur la question des premiers évêques, on consultera avec profit le mémoire de J. Berthollet ${ }^{3}$. Le premier évêque d'Autun fut Rétice, et non Amateur.

La recherche des emplacements successifs des lieux de culte et des établissements ecclésiastiques permet de recueillir un ensemble d'informations sur le développement des premières communautés chrétiennes. On constate de profondes modifications dans la topographie religieuse des cités entre le vir ${ }^{\mathrm{e}}$ et le $\mathrm{Ix}^{\mathrm{e}}$ siècle, provoqués par la fondation de chapitres épiscopaux au début de l'époque carolingienne. C'est seulement alors que la cathédrale prend sa place définitive et que s'organisent les légendes tendant à faire croire qu'elle n'a jamais changé d'emplacement. Avant les servitudes du site, les conditions historiques particulières, l'action d'un évêque contribuent à donner une physionomie particulière à la topographie chrétienne de chaque cité. Il y a cependant des traits communs : la

(1) Du même, ibid., 1943, p. 45-112.

(2) A. LAYER, Recherches historiques sur les Panégyriques de Sidoine-Apollinaire. Paris, 1942, in-8 ${ }^{\circ}$.

(3) J. Berthollet, La question des premiers évêques. Le premier évêque d'Aulun, dans Ann. Bourgogne, 17, 1945, p. 38-44. forme grégaire de ces communautés jusqu'aux $\mathrm{VI}^{\mathrm{e}}-\mathrm{VII}^{\mathrm{e}}$ siècles, les deux groupes de sanctuaires, exlérieur et intérieur, l'exiguitté des lieux de culte, dont on peut conclure à la pauvreté des communautés chrétiennes. Dans la cité, la plus ancienne église est très souvent dans un angle du rempart. Les sanctuaires suburbains ont pour origine une église primitive, avec baptistère et cimetière, près de la route, et souvent cette bourgade chrétienne est plus importante que la cité, dont le rôle économique est des plus restreints 4 .

Les influences qui se sont exercécs sur le plan et la construction des baptistères sont toujours en discussion : Rome ${ }^{5}$, ou les églises d'Asie Mineure ${ }^{6}$.

Des fouilles ont été conduites aux Aliscamps ${ }^{7}$, autour de l'église de SaintHonorat, dans le cimetière in area où trois étages de sépultures ont été reconnus. Les sarcophages sont disposés en accord avec les transformations subies par l'église de Saint-Genès, devenue Saint-Honorat au xir ${ }^{e}$ siècle. A Marseille, la nécropole de Saint-Victor, sur la pente méridionale de la colline donnant sur le Vieux-Port, est sensiblement contemporaine de la fondation de l'abbaye. On constate une absence presque complète de mobiliers funéraires ${ }^{8}$. Un cimetière wisigothique est en cours d'exploration à Sébazac (Aveyron) ${ }^{9}$.

La Guerche est un nom de lieu qui se retrouve souvent dans l'Ouest de la France, pays de Rennes et Nantes. Il conserve le souvenir des fortifications

(4) Jean HUBert, Recherches sur la topographie religieluse des cités de la Gaule du $I V^{\bullet}$ au $I X^{\mathrm{e}}$ siècle, dans $C R A I, 1945$, p. 314-317.

(5) J. Fonmigte, CRAI, 1945, p. 302-307.

(i) M. Aubert, CRAI, 1945, p. 301.

(7) F. BexorT, Gallia, 111, 19.14, p. 257-260.

(8) In même, ibid., p. 260-263.

(9) L. Balsan, Fouilles archéol., 1939-40. 
dressées par les Francs aux frontières bretonnes, du $\mathrm{vi}^{\mathrm{e}}$ au $\mathrm{IX}^{\mathrm{e}}$ siècles, sur une ligne qui ne dépasse pas l'Ille-et-Vilaine et la basse Loire ${ }^{1}$. La cité des Osismi correspondrait à un Finistère agrandi. Dès le ve siècle, Vorgium a son évêque, puis un autre évêque est installé à Aquilona (Locmaria) ${ }^{2}$.

Sur le peuplement germanique en France, deux intéressants travaux sont à méditer. Le premier ${ }^{3}$ est une excellente critique des renseignements fournis par

(1) G. Souillet, Mém. soc. hist. et archéol. Bretagne, XXIV, 1944, p. 24-46.

(2) H. Wacquet, Ann. Bretagne, LII, 1945, p. $56-59$.

(3) F. Lot, CRAI, 1945, p. 829-298. la toponymie; le second ${ }^{4}$ montre que, dans l'Est, les Germains ont été peu nombreux et qu'ils ont été absorbés au cours du $v_{\text {VII }}{ }^{e}$ siècle par l'ancien élément gallo-romain.

Les céramiques trouvées à Curçay (Vienne) n'ont rien d'énigmatique ${ }^{5}$, elles se rattachent aux séries, si nombreuses en Provence, de la poterie wisigothique.

Raymond LANTIER.

Musée des Antiquités nationales, septembre 1946.

(4) E. SAlin, CRAI, 1945, p. 498-504.

(5) Comte de Rilly, Bull. soc. scientif. Ouest, $3^{e}$ sér., XIII, 1944, p. 349-354. 